\title{
Assessing spatial pattern separation in rodents using the object pattern separation task
}

Citation for published version (APA):

van Goethem, N. P., van Hagen, B. T. J., \& Prickaerts, J. (2018). Assessing spatial pattern separation in rodents using the object pattern separation task. Nature Protocols, 13(8), 1763-1792.

https://doi.org/10.1038/s41596-018-0013-x

Document status and date:

Published: 01/08/2018

DOI:

10.1038/s41596-018-0013-x

Document Version:

Publisher's PDF, also known as Version of record

Document license:

Taverne

Please check the document version of this publication:

- A submitted manuscript is the version of the article upon submission and before peer-review. There can be important differences between the submitted version and the official published version of record.

People interested in the research are advised to contact the author for the final version of the publication, or visit the DOI to the publisher's website.

- The final author version and the galley proof are versions of the publication after peer review.

- The final published version features the final layout of the paper including the volume, issue and page numbers.

Link to publication

\footnotetext{
General rights rights.

- You may freely distribute the URL identifying the publication in the public portal. please follow below link for the End User Agreement:

www.umlib.nl/taverne-license

Take down policy

If you believe that this document breaches copyright please contact us at:

repository@maastrichtuniversity.nl

providing details and we will investigate your claim.
}

Copyright and moral rights for the publications made accessible in the public portal are retained by the authors and/or other copyright owners and it is a condition of accessing publications that users recognise and abide by the legal requirements associated with these

- Users may download and print one copy of any publication from the public portal for the purpose of private study or research.

- You may not further distribute the material or use it for any profit-making activity or commercial gain

If the publication is distributed under the terms of Article $25 \mathrm{fa}$ of the Dutch Copyright Act, indicated by the "Taverne" license above, 


\title{
Assessing spatial pattern separation in rodents using the object pattern separation task
}

\begin{abstract}
Nick P. van Goethem ${ }^{1,2}$, Britt T. J. van Hagen ${ }^{1,2}$ and Jos Prickaerts ${ }^{1 \star}$
Pattern separation is the process of transforming highly similar sensory inputs into distinct, dissimilar representations. It takes place in the hippocampus and is thought to be used in episodic memory. Impaired pattern separation performance has been recognized as a predictor for the development of cognitive impairments such as dementia in humans and as being present in patients with schizophrenia and post-traumatic stress disorder (PTSD). In this protocol, we describe how to implement a simple and robust object pattern separation (OPS) task in mice and rats that we have previously established and validated. This two-trial memory task uses specific object locations so differences in performance can be calibrated with the extent of object movement. Changes in performance are indicative of spatial pattern separation. In contrast to other pattern separation tasks, the OPS task allows detection of spatial pattern separation performance bidirectionally. Furthermore, the OPS task is cheaper and easier to use and interpret than other tasks that use more than two objects or that are touch-screen based. The entire protocol, from vivarium acclimatization to training of the animals, takes -35-41 d. After successful training, the animals can be tested repeatedly, and three OPS experiments $(n=20-24$ per experimental day) can be performed per week. A standard level of expertise in behavioral studies in rodents is sufficient to successfully integrate this paradigm into an existing rodent test battery.
\end{abstract}

Introduction

Pattern separation is a memory process that is commonly defined as the ability to form separate representations from highly similar, yet slightly different, events or stimuli. Originating from computational models of hippocampal functioning, pattern separation processes have only recently been shown to be essential for normal memory functioning in both human and animal studies ${ }^{1,2}$. This has led to increased interest in pattern separation as a research topic and, subsequently, a growing body of evidence has emerged to support the importance of pattern separation processes in memory function. Current literature indicates pattern separation performance as a predictor for the development of cognitive impairments, as seen in mild cognitive impairment (MCI), pre-dementia and dementia ${ }^{3}$. Furthermore, pattern separation processes are hypothesized to be impaired in anxiety disorders such as $\mathrm{PTSD}^{2}$. Pattern separation deficits were also reported in schizophrenia patients and have been linked to hippocampal dentate gyrus (DG) dysfunction ${ }^{4,5}$. It has been suggested that impaired pattern separation functioning results in overgeneralization of everyday stimuli and situations, leading to psychotic associations that are likely to underlie the hallmark positive symptomatology of this disorder ${ }^{6}$.

As pattern separation processes have been linked to fundamental hippocampal functioning and different disease models, translatable tests to reliably measure pattern separation performance in rodents are of great interest to both pharmaceutical and fundamental neuroscience research. Different tests to measure pattern separation performance in rodents have been described, for example, fearconditioning paradigms that gradually change context cues $^{2}$, response to three objects in a testing arena $^{7}$, spatial orientation in a radial-arm maze ${ }^{1}$ and response to visual stimuli measured using a touch screen ${ }^{8}$.

Contrasting results have been presented regarding the underlying mechanisms of pattern separation performance. This could be due in part to the lack of consistency between the tasks used in different behavioral in vivo investigations. Because pattern separation takes place on different levels of mnemonic or even sensory processes ${ }^{9,10}$, different paradigms probably measure different underlying

${ }^{1}$ Department of Psychiatry and Neuropsychology, School for Mental Health and Neuroscience, Maastricht University, Maastricht, the Netherlands.

${ }^{2}$ These authors contributed equally: Nick P. van Goethem, Britt T. J. van Hagen. *e-mail: jos.prickaerts@maastrichtuniversity.nl 
neuronal mechanisms. Thus, the fundamental mechanisms of pattern separation and the implications for psychiatric disorders are not fully understood.

In this protocol, we describe a standard protocol for a standardized and simple OPS task. We developed our protocol to be easily implementable in any typical rodent facility and usable by personnel with standard animal behavioral training. Therefore, its use should facilitate a more consistent investigation of spatial pattern separation processes in animal models and treatment intervention studies. Previously, we demonstrated that the OPS can assess the effects of putative pattern separation-enhancing and pattern separation-impairing drugs on OPS task performance ${ }^{11}$.

\section{Development of the OPS task}

We derived our OPS protocol from the popular and straightforward object recognition memory task (ORT) and its spatial variant, the object location task $(\mathrm{OLT})^{12-14}$. On the basis of the same paradigm as the ORT and OLT, the OPS task exploits the naturally occurring exploratory behavior of both rats and mice, which have an innate preference to explore novel stimuli. In the OPS, ORT and OLT tests, the time an animal takes to explore two individual objects in a testing arena is used as a readout parameter (Fig. 1). First, a rodent learns the spatial arrangement of the two objects in a learning trial that consists of 3 or $4 \mathrm{~min}$ of free exploration time. After a 1-h inter-trial interval, the animal is again confronted with the object set, but this time, one of the objects has been moved to a new location,

a

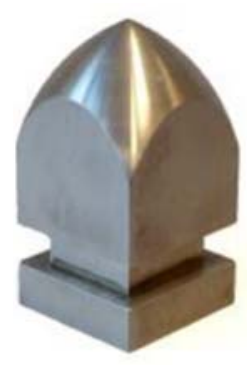

b

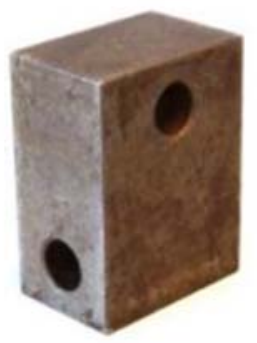

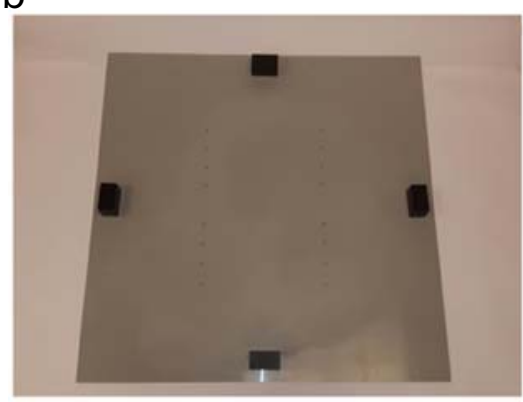

C

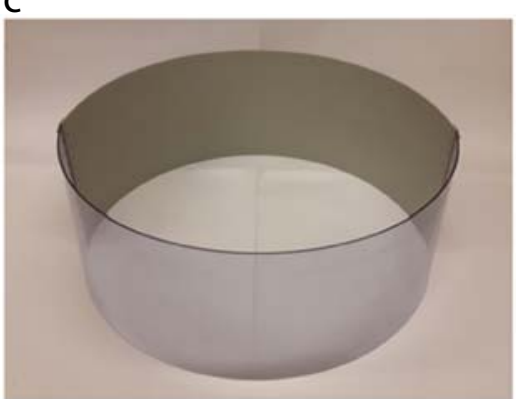

d

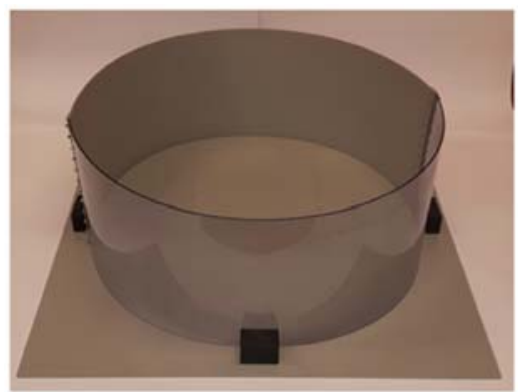

e

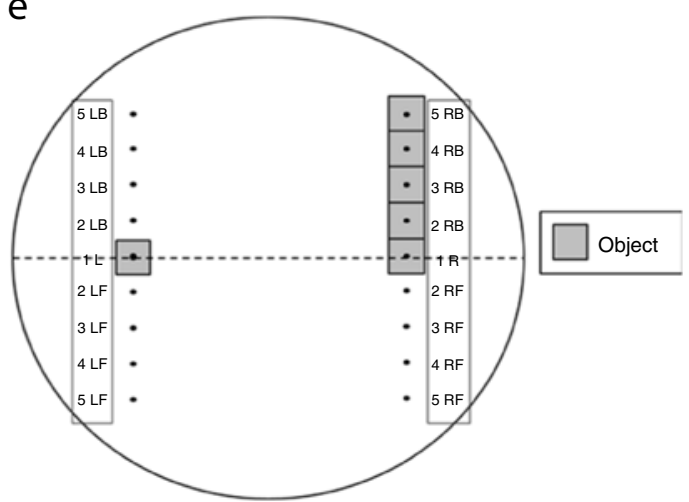

Fig. 1 | Objects, base plate and testing arena used for rats in the OPS paradigm. a, The two different objects used in the OPS task. Both objects are available in duplicate to form the object sets. Usage of the two object sets should alternate between OPS trials. b, The base plate of the OPS apparatus. On this base plate, the testing arena can be placed between the black cubes (which are attached to the base plate); this ensures that the testing arena is always placed on the same location on the base plate (see also d). On the base plate, all possible locations for the objects are indicated with black dots. These dots are $60 \mathrm{~mm}$ (rat base plate) or $45 \mathrm{~mm}$ (mouse base plate) apart and are indicated on both sides (left and right) and directions (forward and backward) of the testing arena (see also e). c, The testing arena; the walls are half-transparent and half-gray or black. d, The testing arena placed on the base plate; for scoring, make sure the experimenter is located in front of the transparent part of the arena wall at an appropriate distance in order to reliably score the exploratory behavior of the animal while not disturbing it. For all exact dimensions of the apparatus and objects, see the 'Materials' section. For mice, similar (although smaller) equipment is used (see 'Materials' section). e, Schematic representation of the top view of the arena, with the possible new locations for an object in T2 (indicated on the right). In the diagram, $\mathrm{L}$ and $\mathrm{R}$ refer to 'left' and 'right', respectively. Furthermore, B and F refer to 'backward' and 'forward', respectively, indicative of the general direction of the displacement of an object. The number indications (1-5) represent the five possible locations to which an object can be displaced. In T1, the placement of objects is always at ' $1 \mathrm{~L}^{\prime}$; in T2, it is always '1R' (after a 1-h interval); one of the objects could be displaced to one of the new locations along the straight line (positions 1-5; see also Box 1). e adapted with permission from ref. ${ }^{15}$, Elsevier B.V. 
which can vary in distance from the location in which it was previously (during the learning trial). Subsequently, it can be determined whether the animal spent a different amount of time exploring the moved or the stationary object. Pattern separation performance is indicated by the relative time spent exploring the moved object as compared with the stationary object. A greater distance between the starting location and the new location should lead to a better pattern separation performance for each rodent, as it is easier to discriminate or recognize the new position as novel when the change is more apparent.

The difference between the OPS task and the OLT is that instead of moving one of the two objects to one new location that is far away from that initially used (which assesses spatial memory performance), the OPS task uses four possible new locations along a vertical axis. These locations gradually increase in separation and range from no displacement (i.e., the same position as in the learning trial) to a maximal displacement similar to that used in the test trial of an OLT $^{15}$ (see also Steps 10-24 of the Procedure, Figs. 1 and 2). Using these subtle differences in locations enables experimenters to find the exact cutoff at which rodents are able to differentiate a new spatial arrangement from an earlier-encountered one. This refined measurement of spatial memory depends on pattern separation processes ${ }^{3}$. When using the smallest possible displacement at which the animals are able to discriminate between the 'old' (learning trial) and 'new' (test trial) situation, it is ensured that the presented locations are highly similar to that presented in the learning trial, and thus pattern separation processes must be used in order to make this discrimination. Another difference and advantage is that the OPS task allows the detection of spatial pattern separation bidirectionally at small spatial separations when using a 1-h inter-trial interval. Thus, in the OPS paradigm, unlike in the OLT, there is a spatial arrangement of the objects that allows detection of improvement or impairment of discrimination performance within the same experiment.

The difference between the OPS task and the ORT is more apparent. The ORT is also a one-trial learning task, but it allows the assessment of consolidation of specifically object information (instead of spatial information) into memory. In the first (learning) trial, an animal is put into an arena in which two identical objects are placed. After a certain delay, the animal is given a second (test) trial, in which it is again placed in the same arena, but now one of the objects has been replaced by a novel object. It is scored by how much time the animal inspects the two objects. Subsequently, it can be determined whether the animal spent a different amount of time on the novel versus the familiar object $^{12-14}$.

\section{Comparison with other pattern separation tasks}

Spatial pattern separation uses memory processes centered in the DG and CA3 region of the hippocampus $^{1,4}$. As such, the OPS task is thought to rely on the capacity to perform hippocampalmediated pattern separation. A comparable spatial task can be easily applied for humans and therefore the OPS task has substantial translational value. In fact, a highly similar spatial pattern separation task for humans has been described and shown to be correlated with performance on the Rey Auditory Verbal Learning Test, which is sensitive to hippocampal dysfunction ${ }^{3}$.

Alternative available pattern separation tasks are often based on fear conditioning ${ }^{2}$ or radial-arm maze ${ }^{1}$ paradigms. Because the OPS task relies on the natural curiosity of rodents, especially for novel stimuli or places, it is not necessary to use positive or negative reinforcement in the form of reward or punishment incentives when using this paradigm. Thus, the OPS task allows measurement of hippocampal-driven pattern separation performance in the absence of any effect on the brain resulting from the punishment and reward incentives. Performance assessment in the OPS task relies on the ability of rodents to discriminate between small spatial changes in object locations within a familiar environment (Figs. 1 and 2). When an object location is recognized by an animal as being different from the one previously encountered, it will naturally spend more time exploring this new object location. Therefore, a difference in exploration times between the displaced and the stationary object in the test trial reflects pattern separation functioning. This is in contrast to pattern separation tasks that use emotional/fear components, which will inherently use a broader range of (pattern separation) processes, as opposed to solely cognitive spatial processes ${ }^{9,10}$.

However, because the spatial nature of the OPS task measures hippocampal-driven pattern separation performance, this can alternatively be interpreted as a limitation of the paradigm when the research question demands measurement of pattern separation on a broader level (i.e., when also 
a

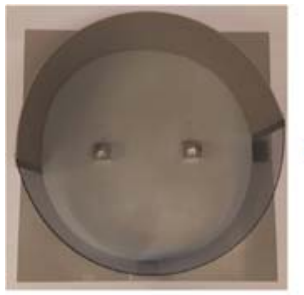

$1 \mathrm{~h}$ )

b

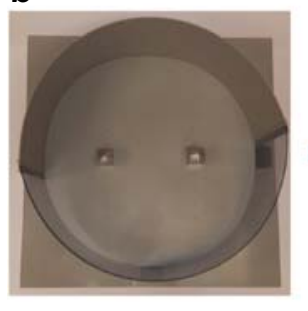

$1 \mathrm{~h}$

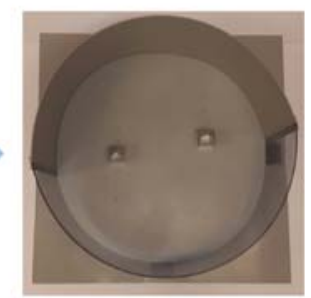

C

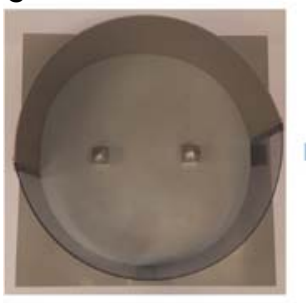

$1 \mathrm{~h}$

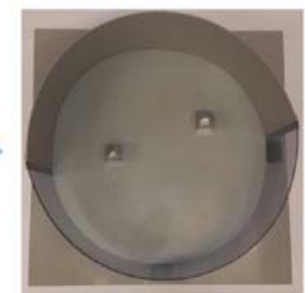

d
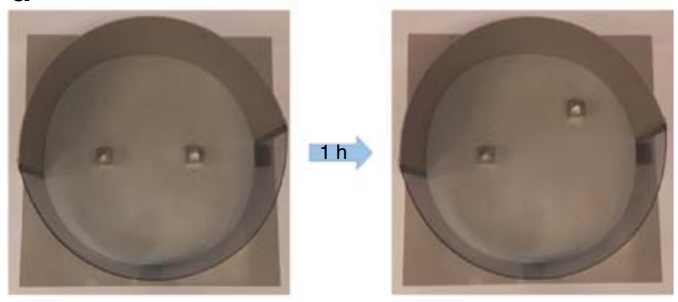

e

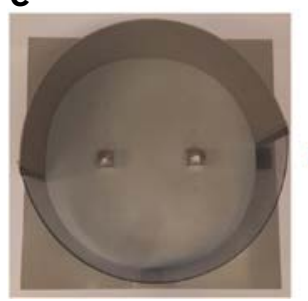

Test trial (T2)

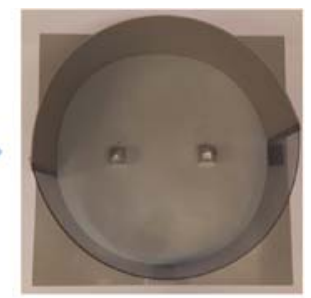

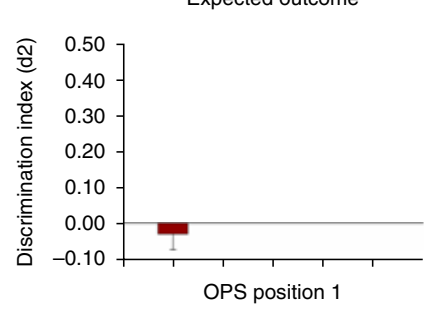

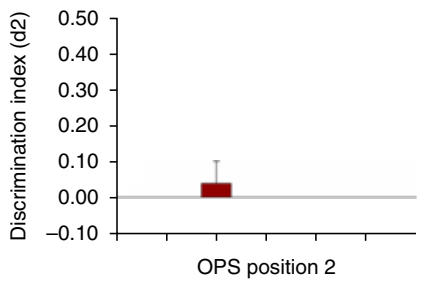

OPS position 2

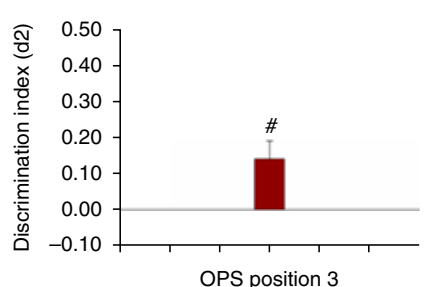

OPS position 3

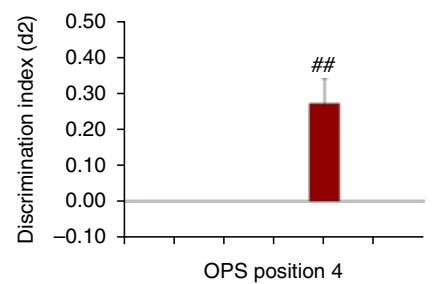

OPS position 4

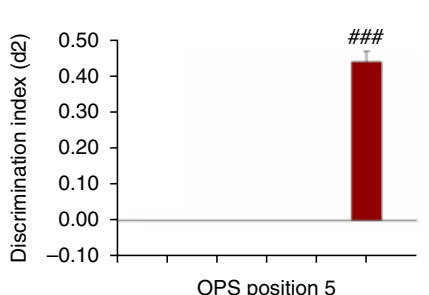

Fig. 2 | An example of object placement in both the learning trial (T1) and the test trial (T2). In this example, one of the similar objects of the object set is moved backward on the right side of the testing arena. The expected outcome of the discrimination index ( $d 2$; see also Table 3) is shown in the graphs in the right column under 'Expected outcome' (mean + s.e.m.). For this example, naive Wistar rats were tested at positions $1(n=13), 2(n=$ 10), $3(n=14), 4(n=10)$ and $5(n=13)$. The data are similar to the data presented in Fig. 4. The intertrial interval was $1 \mathrm{~h}$. Trial duration was 3 min for rats. a, In T1, both objects are placed symmetrically in the middle of the testing arena at the starting positions (position 1). In T2, position 1 is tested. No displacement; this means both objects should be placed symmetrically in the middle of the testing arena again, as in T1. Expected outcome, d2 $\approx$ 0.00. b, For T1, both objects are again placed at position 1. In T2, position 2 is tested. Minimal displacement; expected outcome, d2 $\approx 0.05$. c, For T1, both objects are again placed at position 1. In T2, position 3 is tested. Displacement is halfway, and the expected outcome is d2 $\approx 0.15$. d, For T1, both objects are again placed at position 1 . In T2, position 4 is tested, and the expected outcome is $\mathrm{d} 2 \approx 0.20-0.30$. e, For T1, both objects are again placed at position 1. In T2, position 5 is tested. Displacement is maximal, and the expected outcome is $\mathrm{d} 2 \approx 0.30-0.45$. Significant object location discrimination $(P<0.05)$ performance is found for positions 3, 4 and 5 (or $\mathbf{c}$, $\mathbf{d}$ and $\mathbf{e}$; $t$ test as compared with zero/chance level: ${ }^{\#} P<0.05$; ${ }^{\# \#} P<0.01$

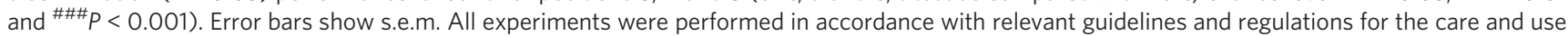
of laboratory animals and were approved by the ethical committee of Maastricht University (protocol: DEC 2012-062). Graphs adapted with permission from ref. ${ }^{11}$, The British Pharmacological Society. 


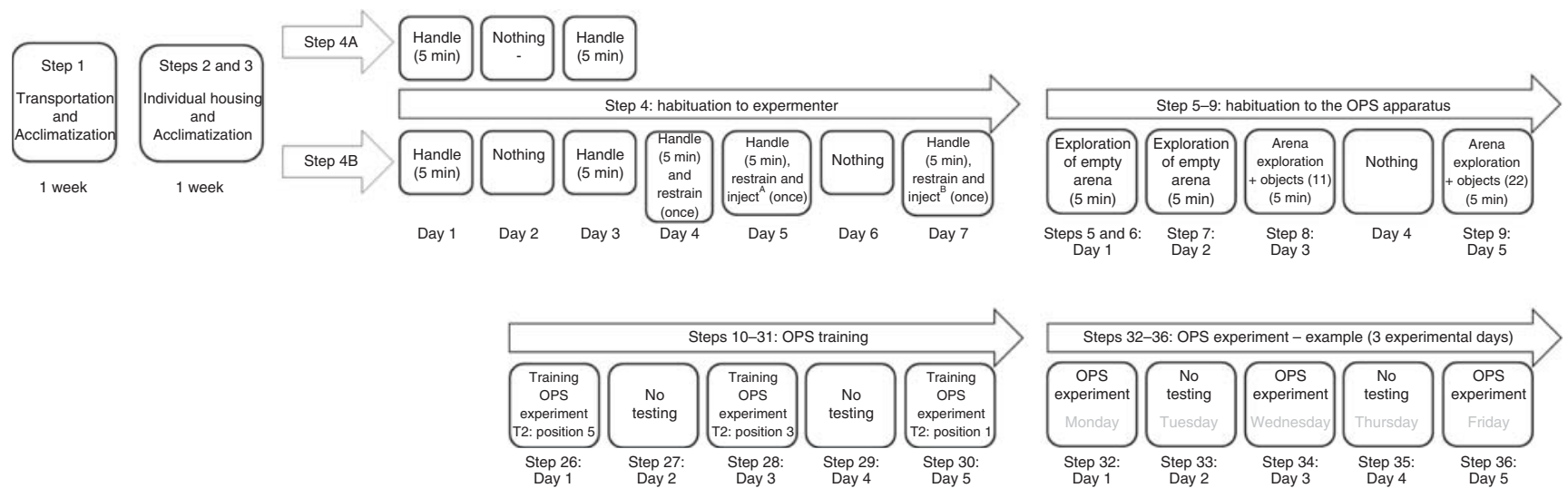

Fig. 3 | Flowchart of the OPS task procedure. This schematic overview shows all steps and timing for OPS training and testing as described in the Procedure.

wishing to assess emotional/fear components). Furthermore, OPS testing requires sufficient arousal, motivation and intact locomotor behavior of the animals in order to have sufficient exploration times for the objects and locations. When assessing the effects of drugs, genetic manipulations, lesions/ ablations or other interventions on pattern separation performance, these aspects should be kept in mind. Interventions that alter the arousal, motivation or locomotor activity of the animals might bias the test results. Moreover, as ORT performance has been shown to be influenced by hormonal regulation in female rats ${ }^{16}$, it is reasonable to suggest that hormonal regulation could affect performance in the OPS paradigm as well. However, we have not yet examined the applicability of female rodents in the OPS task.

Other alternative paradigms have been described that use three or more objects in a testing arena $^{7}$ or that are touch-screen based ${ }^{8}$. Owing to the simple nature and requirements of the setup, the OPS task is less expensive and easier to use and interpret. The simplicity of the task has the additional advantage that no lengthy training procedures are required in order for the assessor to learn how to interpret the rodent strategies or the rules that should be followed when performing a task. The use of different methods for rule and strategy learning is a potential source for inter-study variability.

\section{Experimental design}

To allow for reliable OPS performance and measurement, important parameters for experimenters to consider before and during a study are listed below. Virtually anyone who is licensed to perform animal research and has experience with rodent handling and behavioral tasks can perform the OPS task. No specific level of research expertise or scientific background knowledge is required to follow the OPS protocol. Researchers thinking of implementing the OPS task are advised to adequately teach themselves or technicians how to score exploratory behavior of rodents (see Step 16 of the Procedure), as well-trained experimenters tend to need smaller group sizes and show more consistent results. Behavioral laboratories that already have adequate experience with ORT and/or OLT testing should have no problem implementing the OPS task within their rodent behavioral test battery. The OPS task, OLT and ORT can be performed in the same apparatus, which requires similar habituation and training. This makes the OPS paradigm useful for quick and easy assessment of spatial pattern separation performance, which could potentially lead to more consistent and comparable spatial pattern separation studies across different laboratories.

Before embarking on a study, users must choose and obtain appropriate animals to use as a model, plus set up the required apparatus. Appropriate control groups must be chosen and used in order to minimize the risk of false positives or negatives. Figure 3 provides an overview of the whole procedure. The Procedure itself starts with three preparatory sections: acclimatization (Steps 1-3); habituation (Steps 4-9) and training in the OPS task (Steps 10-31). Once these sections are completed, the animals can be tested repeatedly. Three OPS task experiments can be performed per week, as described in Steps 32-36 of the Procedure. Further OPS experiments can be undertaken on the same animals. If testing is combined with the use of pharmaceutical interventions, the animals can be 


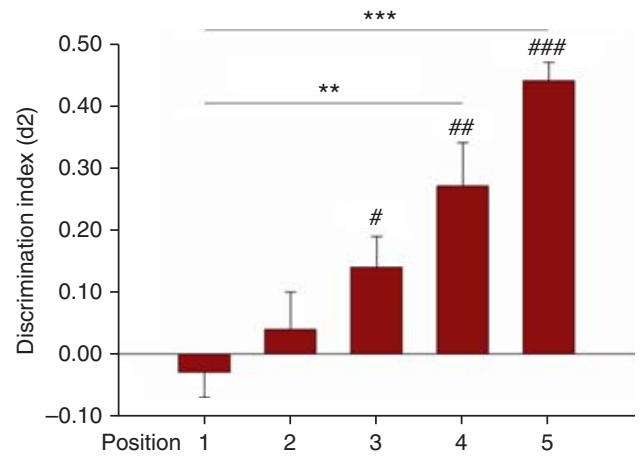

Fig. 4 | Characteristic OPS task results, showing the $\mathrm{d} 2$ indices (mean + s.e.m.) for all five positions of untreated 4-month-old male Wistar rats (average weight $=393 \mathrm{~g})$. Naive Wistar rats were tested on positions $1(n=13), 2(n$ $=10), 3(n=14), 4(n=10)$ and $5(n=13)$. Significant object location discrimination performance was found for positions 3, 4 and 5 ( $t$ test as compared with zero/chance level: ${ }^{\#} P<0.05 ;{ }^{\# \#} P<0.01$ and ${ }^{\# \# \#} P<0.001$ ), which increased with increasing distance from position 1 along the vertical axis (position $3<4<5$ ), which is indicative of spatial pattern separation. When compared with performance on position 1, positions 4 and 5 differed significantly $(P<0.05)$ with respect to pattern separation performance, position 3 showed a trend (one-way ANOVA: $F_{4,55}=$ 13.89; $P<0.001$, post hoc Bonferroni $t$ tests: positions 1 and $3, P=0.1$; positions 1 and $4,{ }^{\star \star} P<<0.01$; and positions 1 and $\left.5,{ }^{\star \star *} P<0.001\right)$. On position 3 , an increase or a decrease in pattern separation performance could be measured (depending on putative pattern separation improving or impairing manipulations, respectively), i.e., performance after an experimental manipulation can resemble the performance at position 5 or 1, respectively. Error bars show s.e.m. Adapted with permission from ref. ${ }^{11}$, The British Pharmacological Society. All experiments were performed in accordance with relevant guidelines and regulations for the care and use of laboratory animals and were approved by the ethical committee of Maastricht University (protocol: DEC 2012-062).

tested repeatedly, providing that sufficient time to allow complete washout of the drug has elapsed between subsequent OPS testing sessions. For some studies, such as lesion/ablation studies, pre- and post-surgery studies can be performed. For studies such as these, post-experimental verification of the correct localization of the brain lesions/ablations should be carried out. Proper memory function of the animals used can be assessed during the OPS training steps (see Steps 25-31 of the Procedure). A characteristic linear OPS task performance (Fig. 4) should result.

\section{Equipment}

Setting up an OPS paradigm within a rodent testing facility requires a limited amount of equipment and expertise. A testing arena, a base plate and objects are required (Fig. 1). Selecting appropriate objects for the rodents to explore is very important. When different object sets are used in the OPS task, it is imperative that both object sets are of equal interest to the rodents. The preference of one object set over another will lead to confounding results in the experiment. To make sure all used object sets are of equal interest to the rodents, pilot tests in which the exploration behavior toward all objects is meticulously assessed should be performed. Equal exploratory behavior/interest in the different objects is important. It has been shown that not all objects are of equal interest to rodents ${ }^{17}$. Furthermore, it is best to use objects that have a square/ rectangular base, in order to properly control the (dis)placement of the objects in the testing arena. As the OPS task is used to assess recognition performance of very small changes in spatial rearrangements, it is imperative that the displacement of the objects be very precise (Figs. 1 and 2). This precise object displacement becomes obscured when objects with a big round base are used, because these are more difficult to precisely displace to the next location in the testing arena when using minimal floor markings.

\section{Choice of animals}

To date, we have validated the OPS task with adult young (2- to 3-month-old) male C57BL/6 mice and Wistar rats. We have found that Wistar rats perform well in this task when using a 1-h inter-trial interval and trial durations of $3 \mathrm{~min}^{11}$. Optimal inter-trial intervals and trial duration for the C57BL/6 mice were found to be 1 or $2 \mathrm{~h}$ and $4 \mathrm{~min}$, respectively. A 30-min inter-trial interval was found to be too short for these mice, i.e., the performance was too high, leading to statistical ceiling effects and an inability to measure the OPS performance bidirectionally ${ }^{15}$. To date, we have not validated the 2 -h inter-trial interval for rats, but we assume this interval could be used as well. As we have not found 
differences in OPS performance between the 1- and 2-h inter-trial intervals in mice (on position 3), we use the 1-h inter-trial interval in our OPS experiments. In addition, we have examined the potential of aged rats (2-year-old male Wistar rats) for use in our OPS paradigm. Our preliminary findings indicate full applicability of aged male Wistar rats in the OPS task using a 1-h inter-trial interval and 3-min trial duration. We have not yet examined the applicability of other rat and mouse strains or rodent species (e.g., hamsters). If variations from expected results occur when testing aged animals or other strains or species, we suggest performing different inter-trial intervals (e.g., $30 \mathrm{~min}$ when the performance is too low, or $3 \mathrm{~h}$ when the performance is too high) or longer trial durations (e.g., $\geq 5 \mathrm{~min}$ when the exploration times are too low) to assess OPS performance. As the OPS task does rely on visual cues, the use of albino animals could be problematic because of the well-known compromised visual acuity of albino animals as opposed to pigmented animals $^{16,18}$. The use of an albino rodent strain might therefore affect certain parameters of behavioral tasks (e.g., the speed of training or discrimination performance). Despite these visual disabilities, the albino Wistar rat strain is often used in (spatial) behavioral paradigms that have led to reproducible results. New animal models should always be carefully assessed before they are used in actual OPS experiments.

It is important to consider the sex of the animals when designing your experiment. It has been shown that the cognitive performance of female rodents fluctuates during the different stages of their estrous cycle ${ }^{16}$. In our experiments to date, we have used only male rats and mice. If female rodents are used in the OPS task, we recommend considering controlling for the estrous cycle (i.e., on the basis of estradiol and progesterone levels). We have validated the OPS task with adult male C57BL/6 mice and Wistar rats ${ }^{11,15}$. Other rodent strains (e.g., for rats: Long-Evans, hooded Lister and Sprague Dawley; for mice: OF1 and NMRI) should also be usable in the OPS paradigm because they exhibit sufficient exploration of the objects ${ }^{16}$. Sufficient object exploration requires adequate energy levels, attention and interest in the objects. These traits may vary between rodent strains ${ }^{16}$. Therefore, we recommend always carefully considering which rodent strain to use in any behavioral paradigm and always controlling for factors that could possibly confound the experimental results. Ways of controlling for these include, for example, performing pilot studies to assess proper performance (see the 'Expected results' section in the Anticipated results) and using different paradigms in order to ensure that the chosen strain is able to perform as anticipated by the experimental design. To elaborate, apart from visual abilities, other possible confounders within an OPS task are the locomotor, arousal and motivational aspects of the animals used. When the OPS task is applied to assess the pattern separation-enhancing or pattern separation-impairing effects of experimental interventions, it should be verified that the observed improvement or impairment in OPS performance is not the result of possible confounding factors related to locomotor, arousal and/or motivational effects of the interventions. Additional tests/separate experiments that specifically assess these traits, before the start of the actual OPS experiments, are recommended. If possible, a within-participants (repeated measures) design is recommended so each animal can serve as its own control. To obtain statistically reliable data, on average, 12-16 animals per group are required, as more animals will strengthen statistical power. Novice experimenters tend to score with higher inter-individual variability and therefore might need bigger group sizes as compared with well-trained experimenters. Thus, we recommend inexperienced experimenters to practice scoring during the habituation phase (Steps 8 and 9).

\section{Animal housing}

Animal housing can be a source of stress for rodents. Care should be taken in regard to these procedures to warrant results that are reproducible and to decrease variation in behavioral data/ performance as much as possible. Upon arrival, reverse the day-night cycle of the animals in the vivarium (e.g., lights off from 7:00 a.m. to 7:00 p.m.). This way, the animals can be tested later on during the day in their active (dark) phase ${ }^{19}$, under low-illumination conditions (i.e., 20 lux, provided by fluorescent red tubes and an indirect background light) (see Step 1 of the Procedure). However, individual housing is preferred (see Step 2 of the Procedure) to ensure that the animals do not have too much interference between the learning and the test trial (see Steps 10-24 of the Procedure). Social housing leads to too much interference during the inter-trial interval and subsequently leads to decreased performance of the animals ${ }^{16,20}$. Individual housing can be a source of stress that in turn may affect the learning abilities, behavioral outcome and biochemical processes of rodents. These could potentially confound the OPS results. Stress and compromised well-being of the animals due to 
individual housing can be assessed by corticosteroid measurements and careful observation of the animals. Before the actual OPS experiments start (see Steps 32-36 of the Procedure), proper cognitive function of the animals must be assessed during the OPS training steps (see Steps 25-31 of the Procedure). With respect to individual housing and isolation stress, it has also been shown that individual housing leads to increased behavioral arousal in mice ${ }^{21}$ and has no effect on stress signs ${ }^{22}$. Nevertheless, to minimize potential bias in the experimental results, acclimatization and habituation to the individual housing is imperative (see Step 2 of the Procedure). Make sure to provide enough cage enrichment and keep this enrichment constant over the entire OPS procedure to avoid other possible interfering factors during the inter-trial intervals. It is important to keep the animals' housing conditions constant during an OPS experiment. Always place the home cage of an animal back at the same spot in the rack and never change the cage enrichment during an experiment. Novel objects in the home cage can lead to interference during the inter-trial interval that can bias the results. The same applies for cleaning the home cages; avoid cleaning or switching the cages on an experimental day (before the OPS experiment), as this could lead to stress and interference during the inter-trial interval $^{23}$. This inter-trial interference also occurs when the animals are socially or group housed ${ }^{20}$ and results in increased variance in the dataset, which can be resolved only by increasing the animal group sizes.

\section{Animal handling}

Experimenter handling is always a source of stress for rodents in experimental studies ${ }^{23}$. Extensive habituation to the handling procedures are therefore imperative to minimize these stress levels (see also Step 4 of the Procedure). Experimenter intervention during the OPS testing can therefore be considered to be a limitation to the paradigm. To minimize experimenter handling, clever designs can be found in the literature, in which a behavioral apparatus is used that requires only minimal animal handling due to use of holding areas within the testing apparatus. During the inter-trial interval, an animal can stay within this holding area until the next trial starts. This minimizes experimenter handling and also offers the possibility to make use of a continuous-running protocol ${ }^{24-27}$. However, this does require an animal to stay within this holding area for the total duration of the inter-trial interval (which is $1 \mathrm{~h}$, in the case of the OPS task). Hence, to adapt such a behavioral apparatus to the OPS task would require modifications to keep the testing schedules workable (e.g., making use of multiple holding areas/chambers so that multiple animals can be tested in sequence or use of multiple apparatuses). Habituation sessions to familiarize the animals to the experimenter and the handling procedures should be performed carefully (see Step 4 of the Procedure). Failure to habituate the animals properly could lead to stressed animals and biased results. The whole idea of this procedure is to let the rodents become accustomed to the smell and handling of the experimenter. Proper habituation to handling will greatly benefit the experimenter later on when the animals must be handled regularly during testing and/or possible administration of substances. Ideally only one experimenter should be assigned to an animal cohort. This person should habituate the animals to the handling procedure and also perform the OPS experiment. Switching between experimenters is a cause of stress for rodents and will bias your experimental results ${ }^{28}$. Be gentle with the rodents and avoid restraining them too much. Restraining the animals will lead to animals that are even more difficult to handle later on. Animals that do not habituate to handling well should be given a little more time to become accustomed to the handling procedure. Never pick up a rodent at the tip of the tail, as this may cause severe skin injury and stress (see Step 4 of the Procedure). Handling on additional days without restraining and/or injecting the animals will attenuate their stress levels during subsequent handling. Consider weighing your animals on separate days from the OPS protocol to increase low-stress contact moments.

\section{Timing of experiments}

As rodents are nocturnal species, it is important to carefully consider the timing of the OPS experiments. Nocturnal species are more active during the dark phase, so testing during the light (resting) phase is likely to result in behavioral differences, behavioral inhibition and/or cognitive disruption $^{19}$. For this reason, we typically test our rodents in all OPS task experiments during the dark/active phase. The OPS task does rely on visual cues. Therefore, the task should be performed in low-light conditions. The lighting conditions should be $\sim 20$ lux and should be equal in the different parts of the testing arena. After habituation to the reversed day-night cycle, we can test our animals in the daytime during their active (dark) phase (e.g., between 7:00 a.m. and 7:00 p.m.). Make sure to 
allow enough time for the animals to become acclimated to the reversed day-night cycle (at least 2 weeks; see also Steps 1-4 of the Procedure) in order for their circadian rhythm to adjust. In addition to the impact of light, the behavioral outcome of rodents is also dependent on their circadian rhythms ${ }^{19}$. Consequently, when testing repeatedly, it is advised to conduct the OPS experiments at the same time on each experimental test day. Finally, in regard to rodent caretaking, it is important to consider when the animals' cages are cleaned. Cage cleaning is quite stressful for rodents ${ }^{23}$ and should therefore never occur right before an experiment. Plan the cleaning/changing of the cages either after the experiment or on a day when no testing occurs.

\section{Testing environment}

The housing and testing conditions of the rodents largely depend on the availability of space in the animal facility. Ideally, the animals should be housed in the same room as that used for OPS testing. As this is not always an option, careful consideration must be made when the animals are housed in a different room from that where the testing will take place (see Step 3 of the Procedure). To warrant reliable and reproducible results, the following parameters should be considered.

When housing and testing occur within the same room, make sure the placement of the cages will not interfere with the behavior of the animals within the testing arena. Ideally, the animal cages should be placed on the right (50\% of animals) and left (50\% of animals) side of the testing arena at a sufficient distance (as far away from the testing arena as possible). This way, potential side preferences of the animals within the testing arena should be eliminated. For example, because of the unavoidable noises (and odors) coming from the side on which the home cages are placed, the animal that is being tested can become distracted and prefer this side of the arena. As the OPS task is based on spatial arrangement of objects within the testing arena, this may confound the results. If the animals are housed on both the left and right side of the testing arena, this potential problem will be eliminated. Another possibility is to house the animals at the front and back of the testing arena. It is less likely that there will be issues with larger rooms . It is always wise to check for side preferences (left and right/front and back) to assess whether everything in your behavioral room is placed in a way that avoids side preferences (see also Anticipated results).

When the rodents are housed in a different room from that in which they are tested, they must be transported to the testing room for the actual experiment. Ideally, the rodent cages will be permanently placed in movable racks so the animals can be transported easily, with the least amount of

Table 1 | Randomization schemes of objects and locations for OPS testing at positions 1, 3 and 5

\begin{tabular}{|c|c|c|c|c|c|c|c|}
\hline \multirow[b]{2}{*}{$\begin{array}{l}\text { Animal } \\
\text { number }\end{array}$} & \multirow[b]{2}{*}{ Objects } & \multirow{2}{*}{$\begin{array}{l}\text { Learning trial (T1) } \\
\text { Position of } \\
\text { stationary objects }\end{array}$} & \multicolumn{5}{|c|}{ Test trial (T2) } \\
\hline & & & $\begin{array}{l}\text { Object moved } \\
\text { left backward }\end{array}$ & $\begin{array}{l}\text { Object moved } \\
\text { left forward }\end{array}$ & $\begin{array}{l}\text { Object moved } \\
\text { right backward }\end{array}$ & $\begin{array}{l}\text { Object moved } \\
\text { right forward }\end{array}$ & $\begin{array}{l}\text { New position } \\
\text { of the object }\end{array}$ \\
\hline 1 & 22 & 1 & NA & & & & 1 \\
\hline 2 & 11 & 1 & & & $x$ & & 3 \\
\hline 3 & 22 & 1 & $x$ & & & & 5 \\
\hline 4 & 11 & 1 & NA & & & & 1 \\
\hline 5 & 22 & 1 & & $x$ & & & 3 \\
\hline 6 & 11 & 1 & & & & $x$ & 5 \\
\hline 7 & 11 & 1 & NA & & & & 1 \\
\hline 8 & 22 & 1 & & & $x$ & & 3 \\
\hline 9 & 11 & 1 & $x$ & & & & 5 \\
\hline 10 & 22 & 1 & NA & & & & 1 \\
\hline 11 & 11 & 1 & & $x$ & & & 3 \\
\hline 12 & 22 & 1 & & & & $x$ & 5 \\
\hline
\end{tabular}

Randomization scheme for 12 animals to be tested at positions 1, 3 and 5 in the OPS task. From left to right, the columns show 'Animal nr.', this corresponds to the ID numbers of the animals; 'Objects', refers to the object set, here the sets of two objects are called '11' (object set 1) and '22' (object set 2); 'Learning trial (T1)', shows the position the objects should be at during the first trial (T1); this is always position 1; the column 'Test trial (T2)' indicates the new location (side, direction and position) to which one object should be moved. The subcolumns show whether the right or the left object must be moved, to which position and in what direction (backward or forward) this object must move. 'Position' indicates the position to which the object must be displaced. The ' $X$ ' indicates where the object must be in T2, e.g., rat 2 obtains object set 11 , in T1 both objects are placed symmetrically at position 1 on both the left and the right side of the testing arena and in T2 the right object must be moved backward to position 3. As there is no displacement when testing position 1 in T2, the 'Moved object' is indicated as 'NA' when position 1 is used in T2. 
Table 2 | Randomization schemes of objects and locations for OPS testing at position 3

\begin{tabular}{|c|c|c|c|c|c|c|c|}
\hline \multirow[b]{2}{*}{$\begin{array}{l}\text { Animal } \\
\text { number }\end{array}$} & \multirow[b]{2}{*}{ Objects } & \multirow{2}{*}{$\begin{array}{l}\text { Learning trial (T1) } \\
\text { Position of } \\
\text { stationary objects }\end{array}$} & \multicolumn{5}{|c|}{ Test trial (T2) } \\
\hline & & & $\begin{array}{l}\text { Object moved } \\
\text { left backward }\end{array}$ & $\begin{array}{l}\text { Object moved } \\
\text { left forward }\end{array}$ & $\begin{array}{l}\text { Object moved } \\
\text { right backward }\end{array}$ & $\begin{array}{l}\text { Object moved } \\
\text { right forward }\end{array}$ & $\begin{array}{l}\text { New position } \\
\text { of the object }\end{array}$ \\
\hline 1 & 11 & 1 & & $x$ & & & 3 \\
\hline 2 & 22 & 1 & & & $x$ & & 3 \\
\hline 3 & 11 & 1 & $x$ & & & & 3 \\
\hline 4 & 22 & 1 & & & & $x$ & 3 \\
\hline 5 & 11 & 1 & & $x$ & & & 3 \\
\hline 6 & 22 & 1 & & & $x$ & & 3 \\
\hline 7 & 22 & 1 & $x$ & & & & 3 \\
\hline 8 & 11 & 1 & & & & $x$ & 3 \\
\hline 9 & 22 & 1 & & $x$ & & & 3 \\
\hline 10 & 11 & 1 & & & $X$ & & 3 \\
\hline 11 & 22 & 1 & $x$ & & & & 3 \\
\hline 12 & 11 & 1 & & & & $x$ & 3 \\
\hline
\end{tabular}

Randomization scheme for 12 animals to be tested only at position 3 in the OPS task. For additional explanation, see footnote in Table 1. Object location discrimination performance at position 3 can be used to assess pattern separation bidirectionally, i.e., improved or impaired performance (e.g., for drug testing or model validation, respectively).

stress. Make sure to habituate the animals to this transportation procedure, as it may cause stress the first few times they are moved and placed in another room (e.g., perform this procedure twice before actual handling). Before handling (see Step 4 of the Procedure) or testing (see Steps 10-36 of the Procedure) the animals, leave them undisturbed for at least $30 \mathrm{~min}$ in order for them to become acclimatized to the environment.

If a dedicated room for OPS testing is available, the base plate, testing arena and OPS objects can remain untouched until the next testing session. In addition, all other things in the room should remain similar (e.g., desk, PC). This way, the same spatial arrangement of the room is ensured over subsequent test days, which is preferable when performing any behavioral rodent task related to spatial learning. Store the OPS objects that are not being used during the learning/test trial out of sight of the animals in the testing arena (e.g., under the table on which the testing arena is placed or behind the testing arena on the side of the gray/black half of the arena wall). If the testing room is used for different behavioral tests by multiple persons, the experimenter must make sure that the testing conditions and layout of the room are exactly replicated over subsequent experimental days. Changes in the environment may cause variability in the behavioral outcomes.

\section{Habituation to the OPS procedure}

After the rodents are habituated to the handling by the experimenter (see Step 4 of the Procedure), they must become habituated to the OPS testing apparatus (see Steps 5-9 of the Procedure). First, the animals can become familiarized with the testing arena on two separate days. Subsequently, the animals can become familiarized with the OPS objects within the testing arena again on two separate days. It is important to habituate/familiarize the animals with the sets of objects that are going to be used. Using novel objects on each experimental day is difficult to achieve if multiple testing sessions are being used (i.e., many equally interesting objects must be found and used). The OPS task is designed for repeated testing, and therefore the animals should become familiarized with the used objects so the pattern separation performance resembles an 'update' of the memory trace ${ }^{29}$ (in contrast to reflecting absolute novelty ${ }^{30}$ ). If properly habituated, the animals can be tested for at least ten times, given that the two sets of objects are alternated between testing days.

When using mice, in order to increase their arousal at testing, they are placed in an empty cage (only bedding, no enrichment) for 4 min immediately preceding the trials in OPS experiments. This will increase the exploratory behavior of the mice during testing ${ }^{15,31}$. In our lab, we add this extra procedure only when using mice, as rats show sufficient exploratory behavior when placed immediately from the home cage into the testing arena. Therefore, when using mice, the extra procedure of making such an 'incubation cage' should be taken into account when preparing the testing room. Mice should become accustomed to the empty cage and the procedure (during 


\section{Box 1 | Positioning of the OPS objects}

Position 1: No displacement. The placement of the objects is exactly the same as during T1. Therefore, an improvement on position 1 is impossible, as the conditions in $T 1$ and $T 2$ are exactly the same. The $d 2$ value should be 0.00 (i.e., chance level) or should at least not significantly differ from zero $(P>0.05)$. Position 1 serves as a control, as no pattern separation can or should be detected here.

Position 2: At position 2, the displacement of one of the objects is very minimal, and rodents normally show no to only very slight pattern separation performance at this position. When an improvement is found at position 3 within a certain experimental condition, the experimenter could consider testing the experimental condition at position 2 as well. By experimentally increasing pattern separation performance at position 3 , this performance might increase at position 2 as well. Therefore, position 2 can be used to further investigate or refine the improved performance within an experimental condition. If no effect or even an impairing effect is found at position 3, the experimenter should consider increasing the displacement to position 4 (i.e., when an impairment in performance is found at position 3, it should also be present at position 2). The $\mathrm{d} 2$ value should be $\sim 0.05$ or slightly above chance level. The $\mathrm{d} 2$ value will not significantly differ from zero/chance level $(P>0.05)$ in naive/ untreated animals.

Position 3: At position 3, the displacement of one object is exactly halfway between the displacements at positions 1 and 5. This is the most relevant position for testing experimental conditions. The $\mathrm{d} 2$ value should be $\sim 0.15$ and significantly differ from zero/chance level $(P<0.05)$ and could show a trend or a significant difference $(P<0.05)$ from performance at position 1 (ref. 15). A significant difference from chance level $(P<0.05)$ indicates that the rodents perceive the displaced object as being in a 'novel' location, meaning they show functioning spatial pattern separation. At position 3, performance can vary bidirectionally, i.e., the d2 value can either decrease or increase (as compared with baseline, $\mathrm{d} 2 \approx 0.15$ ), depending on the experimental condition that is tested. For example, in drug testing, a pattern separation-impairing drug will decrease the $d 2$ value (e.g., from 0.15 to 0.00 ), whereas a pattern separation-improving drug will increase the $\mathrm{d} 2$ value (e.g., from 0.15 to $0.40)$. This way, a pattern separation-improving drug can show 'position 5 performance' $(\mathrm{d} 2 \approx 0.40)$ at position 3 , whereas a pattern separation-impairing drug can show 'position 1 performance' ( $d 2 \approx 0.00$ ) at position 3 (ref. 11). The same holds for, e.g., lesion studies, food-deprivation studies and genetic manipulations.

Position 4: Position 4 is located between position 3 and position 5 . Similar to position 2, position 4 is considered to be a measure that can be used to further investigate or refine the performance found at position 3 . When testing an experimental condition and no or only a slight effect is found at position 3, the experimenter could consider testing the experimental condition at position 4 to see whether performance there is enhanced as compared with baseline (i.e., the control group). Testing at position 4 when an improvement has already been found at position 3 would not be very informative, because of ceiling effects (i.e., when an improvement in performance has already been found at position 3, it should also be present at position 4). The $\mathrm{d} 2$ value should be between 0.20 and 0.30 and should significantly differ from zero/chance level and performance at position $1(P<0.05)$.

Position 5: This position represents maximal displacement. At position 5, the performance cannot go higher, as the displacement is maximal here. Therefore, at position 5 a ceiling effect is encountered (i.e., OPS performance cannot improve any further). The only pattern separation effect (or spatial memory effect) that can be measured at position 5, with a 1-h inter-trial interval, is performance impairment (similar to the $\mathrm{OLT}^{32}$ ). The $\mathrm{d} 2$ value should be between 0.30 and 0.45 in naive/untreated animals and should significantly differ from zero/chance level and performance at position $1(P<0.05)$.

\section{Table 3 | Derived measures in the object pattern separation task}

Trial number

Exploration time (s)
Discrimination index

$$
\mathrm{e} 1=\mathrm{a} 1+\mathrm{a} 2
$$$$
\mathrm{e} 2=\mathrm{a} 3+\mathrm{b}
$$

Not applicable

$\mathrm{d} 2=(\mathrm{b}-\mathrm{a} 3) / \mathrm{e} 2$

$\mathrm{e} 1$ is the time spent exploring the two identical object locations (a1 and a2) during the learning trial (T1). e2 is the time spent in exploring both the familiar (a3) and new object location (b) in the test trial (T2). d2 corresponds to the ability to discriminate between the old and new object location during the test trial (T2). The $\mathrm{d} 2$ index is corrected for exploration time during $\mathrm{T} 2$ and is therefore independent of the animals' exploratory behavior during this trial.

Steps 8 and 9 of the Procedure) 4 min before they go into the testing arena with the two similar objects. The bedding material should be changed after testing each cohort of animals. This allows the bedding to be soiled in a similar manner when the animals are tested in the same order on each habituation day. Alternatively, an empty cage without bedding could be used, but make sure to clean this cage after each animal. This will be more time consuming, as the cage must be cleaned with an ethanol solution and the ethanol must be evaporated completely before a new mouse can be put in the empty cage. 
Table 4 | Testing schedule for two OPS experiments using mice in the presence or absence of injection

\begin{tabular}{|c|c|c|c|c|c|}
\hline Time & Exp $1 \mathrm{~T} 1$ & Exp 1 T2 & Exp 2 Inj. & $\operatorname{Exp} 2 \mathrm{~T} 1$ & Exp $2 \mathrm{~T} 2$ \\
\hline 0:00 & 1 & & 1 & & \\
\hline $0: 06$ & 2 & & 2 & & \\
\hline $0: 12$ & 3 & & 3 & & \\
\hline $0: 18$ & 4 & & 4 & & \\
\hline $0: 24$ & 5 & & 5 & & \\
\hline $0: 30$ & 6 & & 6 & 1 & \\
\hline $0: 36$ & 7 & & 7 & 2 & \\
\hline $0: 42$ & 8 & & 8 & 3 & \\
\hline $0: 48$ & 9 & & 9 & 4 & \\
\hline $0: 54$ & 10 & & 10 & 5 & \\
\hline 1:00 & & 1 & & 6 & \\
\hline 1:06 & & 2 & & 7 & \\
\hline 1:12 & & 3 & & 8 & \\
\hline 1:18 & & 4 & & 9 & \\
\hline $1: 24$ & & 5 & & 10 & \\
\hline 1:30 & & 6 & & & 1 \\
\hline 1:36 & & 7 & & & 2 \\
\hline 1:42 & & 8 & & & 3 \\
\hline $1: 48$ & & 9 & & & 4 \\
\hline $1: 54$ & & 10 & & & 5 \\
\hline $2: 00$ & & & & & 6 \\
\hline $2: 06$ & & & & & 7 \\
\hline $2: 12$ & & & & & 8 \\
\hline $2: 18$ & & & & & 9 \\
\hline $2: 24$ & & & & & 10 \\
\hline
\end{tabular}

The timing information is in the format hour:minute. The numbers in the tables correspond to the individual animal numbers. $\mathrm{T} 1$ and $\mathrm{T} 2$ are the learning trial and the testing trial, respectively, of the OPS task. The number of the animal to be injected at each time point is indicated in the 'Inj.' column. This is an OPS test schedule for mice that must be injected with a drug that has a $T_{\max }$ of $30 \mathrm{~min}$ and must be maximally active during the memory-acquisition phase (i.e., the animal must be injected 30 min before the learning trial T1).

\section{Planning for OPS testing}

The animals are familiarized with the OPS objects during habituation to OPS testing (Steps 8 and 9 of the Procedure). During the actual OPS testing, the rodents' reactions to changes in the position of the OPS objects are monitored. Thus, the precise positioning of the objects for each rodent and each test must be determined before starting the OPS task, using a randomization schedule (discussed further in the 'OPS testing' section; see also Tables 1 and 2). Objects are displaced to one of five different positions, numbered 1-5 along a vertical axis. These positions are indicated on the base plate of the OPS apparatus (Fig. 1). The most straightforward way of measuring pattern separation is by first establishing performance at positions 1, 3 and 5. Figures 1 e and 2 show the various possible positions in the arena visually and Box 1 lists what use of each position means.

\section{OPS testing}

Animals undertake the OPS task during training (Steps 10-24 of the Procedure), performance measurement (Steps 25-31 of the Procedure) and the actual tests (an example is described in Steps 32-36 of the Procedure). The OPS task is a two-trial spatial learning paradigm. The task starts with the first trial, which is called the learning trial or T1. After T1, there is a second trial $(1 \mathrm{~h}$ after the end of T1), which is called the test trial or T2. The object exploration times of this trial are used to calculate the discrimination index $(\mathrm{d} 2)$ to assess the pattern separation performance of the animals (see Anticipated results, Table 3).

Only relatively short inter-trial intervals (i.e., 1 or $2 \mathrm{~h}$ ) can be used in the OPS task in order to reliably measure spatial pattern separation bidirectionally ${ }^{15}$. Using longer inter-trial intervals within the OPS 
Table 5 | Testing schedule for two OPS experiments using rats in the presence or absence of injection

\begin{tabular}{|c|c|c|c|c|c|}
\hline Time & Exp $1 \mathrm{~T} 1$ & Exp $1 \mathrm{~T} 2$ & Exp 1 Inj. & Exp $1 \mathrm{~T} 1$ & Exp $1 \mathrm{~T} 2$ \\
\hline $0: 00$ & 1 & & 1 & & \\
\hline 0:05 & 2 & & 2 & & \\
\hline 0:10 & 3 & & 3 & & \\
\hline $0: 15$ & 4 & & 4 & & \\
\hline $0: 20$ & 5 & & 5 & & \\
\hline $0: 25$ & 6 & & 6 & & \\
\hline $0: 30$ & 7 & & 7 & 1 & \\
\hline $0: 35$ & 8 & & 8 & 2 & \\
\hline $0: 40$ & 9 & & 9 & 3 & \\
\hline $0: 45$ & 10 & & 10 & 4 & \\
\hline $0: 50$ & 11 & & 11 & 5 & \\
\hline $0: 55$ & 12 & & 12 & 6 & \\
\hline 1:00 & & 1 & & 7 & \\
\hline 1:05 & & 2 & & 8 & \\
\hline 1:10 & & 3 & & 9 & \\
\hline 1:15 & & 4 & & 10 & \\
\hline $1: 20$ & & 5 & & 11 & \\
\hline $1: 25$ & & 6 & & 12 & \\
\hline 1:30 & & 7 & & & 1 \\
\hline 1:35 & & 8 & & & 2 \\
\hline $1: 40$ & & 9 & & & 3 \\
\hline 1:45 & & 10 & & & 4 \\
\hline 1:50 & & 11 & & & 5 \\
\hline 1:55 & & 12 & & & 6 \\
\hline $2: 00$ & & & & & 7 \\
\hline 2:05 & & & & & 8 \\
\hline $2: 10$ & & & & & 9 \\
\hline 2:15 & & & & & 10 \\
\hline $2: 20$ & & & & & 11 \\
\hline $2: 25$ & & & & & 12 \\
\hline
\end{tabular}

The timing information is in the format hour:minute. The numbers in the tables correspond to the individual animal numbers. $\mathrm{T} 1$ and $\mathrm{T} 2$, are the learning trial and the testing trial, respectively, of the OPS task. The number of the animal to be injected at each time point is indicated in the 'Inj.' column. This OPS test schedule is for rats that must be injected with a drug that has a $T_{\max }$ of $30 \mathrm{~min}$ and must be maximally active during the memory-acquisition phase (i.e., the animal must be injected 30 min before the learning trial $\mathrm{T} 1$ ).

paradigm leads to statistical floor effects. At longer inter-trial intervals, small spatial separations are not remembered by the rodents, leading to a need to increase the spatial separation to a level that could arguably require (normal) long-term spatial memory instead of pattern separation processes. Having to use a short inter-trial interval (i.e., 1 or $2 \mathrm{~h}$ ) results in a potential limitation of the OPS paradigm when the researcher wants to differentiate between the different phases of memory functioning (i.e., the memory acquisition, consolidation and retrieval processes). The action of a certain intervention is likely to overlap the different phases of memory functioning when using a short inter-trial interval. Therefore, we use the OPS task as a paradigm to measure spatial pattern separation per se, as opposed to that of a particular acquisition, consolidation or retrieval memory process.

The duration of both $\mathrm{T} 1$ and $\mathrm{T} 2$ is $3 \mathrm{~min}$ for rats $^{11}$ and 4 min for mice $\mathrm{e}^{15}$. This trial duration is in line with earlier studies using object recognition or object location as a primary readout ${ }^{16,32}$. Although short, we have found robust pattern separation performance when using this trial duration ${ }^{11,15}$ (Fig. 4). Having a longer trial duration for mice (4 min) helps to increase their exploration time and discrimination performance. Therefore, when planning an OPS experiment, it is feasible to allocate a 5-min window for rats ( 5 min per rat), and a 6-min window for mice (6 min per mouse) (Tables 4 and 5). With a 3-min (rat) and a 4-min (mouse) trial duration, 2 min will remain, which can be used for cleaning, changing objects and handling animals (as well as optional administration of drugs). 
Careful analysis of a large dataset of ORT results has shown reliable object discrimination performance (i.e., a stabilized discrimination index (d2), see also the 'Statistical analysis' section of the Anticipated results) when familiarized rats manifested a total exploration time of minimally $7 \mathrm{~s}$ in $\mathrm{T} 1$ and $10 \mathrm{~s}$ in T2. Although these minimal exploration times for both objects seem low, apparently, rats are able to learn object features within this relatively short time frame ${ }^{30}$. Even though the ORT is not a spatial task, this cutoff for total exploration time has been applied to the OLT in familiarized animals as well (both for rats and mice) ${ }^{32,33}$. Our results indicated that a similar cutoff can be applied to the OPS task. Hence, we believe that in order for the animals to properly learn the spatial arrangement of the objects in the testing arena, a cutoff in minimal exploration time similar to that found for the ORT can be used (i.e., $T 1 / e 1 \geq 7 \mathrm{~s}$ and $T 2 / e 2 \geq 10 \mathrm{~s}$ (where $e 1$ is the total exploration time in the first/learning trial (T1) and $e 2$ is the total exploration time in the second/test trial (T2)) for familiarized animals ${ }^{30}$ ) (see Steps 10-24 of the Procedure). We rarely encounter nonperformers in the OPS task when using either male Wistar rats or male C57BL/6 mice. When encountered, this probably relates to elevated anxiety and/or stress levels, which could be counteracted by proper habituation of the animals to the test and handling procedures. Alternatively, using different intertrial intervals (e.g., $30 \mathrm{~min}$ when the performance is too low or $3 \mathrm{~h}$ when the performance is too high) or longer trial durations (e.g., $\geq 5$ min when the exploration times are too low) could help minimize the number of nonperformers. Animals that do show a lower exploration time in one or both of the OPS trials should be removed from the dataset.

The randomization scheme should be designed so that both the positions to be tested in T2 and the objects used are assigned in a balanced manner to prevent potential biases due to preferences for particular locations or objects. An example is given for 12 animals in the appended randomization scheme, in which positions 1, 3 and 5 (Table 1) or position 3 only (Table 2) are tested in a balanced way with two objects. Trained animals must be assigned (pseudo) randomly to experimental groups to warrant reliable results (e.g., see Tables 1 and 2). Moreover, if applicable, the experimenter should always be blinded to the treatment condition of the animals.

Materials

\section{Reagents}

- Rodents: For the assessment of spatial pattern separation performance using the OPS task, both commercially available or in-house laboratory-bred rodents can be used. In our experiments, we normally use adult male Wistar rats (2-3 months old) or adult male C57BL/6 mice (2-3 months old). Both the Wistar rats and the C57BL/6 mice we use are ordered from Charles River (Wistar rat: Crl:WI, strain code 003; C57BL/6 mouse: C57BL/6NCrl, strain code 027). These rodent strains are known to show robust performance in memory tasks regarding object recognition or location performance ${ }^{16,32}$. ! CAUTION All experiments performed on rodents must be performed in accordance with relevant governmental guidelines and regulations after being approved by a local ethical committee for the care and use of laboratory animals. All procedures should be designed and performed to minimize the potential discomfort of the animals during the behavioral experiments. Our experimental procedures are in strict accordance with the Dutch Experiments on Animals Act (EAA, amended in 1996) and the European Directive (2010/63/EU) of the European Parliament and of the Council of the European Union (86/609EEC) on the protection of animals used for scientific purposes (22 September 2010), and were approved by the local ethical committee for animal experiments of Maastricht University. Maastricht University has a license to perform animal experiments (license ex art 2, 1 and art6 EAA: DGVGZ/VVP-83267). The detailed project proposal for the studies conducted to validate the hereindescribed OPS protocol was approved after careful evaluation by an ethical committee of Maastricht University (license animal ethical committee: Min.VWS, GZBIVVB 981845, 16 April 1998). The assigned official protocol numbers provided to these studies were DEC 2012-062 and DEC 2013-013, for the rat studies, and DEC 2013-059, for the mouse studies.

- Ethanol (70\% (vol/vol); Boom B.V., cat. no. 84010059.2500)

- Laboratory animal bedding (Rehofmix corncob bedding for rodents MK1500; Tecnilab-BMI, cat. no. 97150)

- Rodent chow in pellet form (rat/mouse maintenance, $10 \mathrm{~mm}$, sterilized, $25 \mathrm{kGy}, 10-\mathrm{kg}$ bag; SSNIFF, cat. no. V1534-703) $\Delta$ CRITICAL Store chow appropriately, ensuring hygienic, cool and dry conditions. The optimum storage temperature is $\leq 18{ }^{\circ} \mathrm{C}$. Closed bags can be stored until expiration date. Open bags should be used within 6 weeks if they have been properly stored. 


\section{Equipment}

- Animal housing (Equipment setup)

- Water bottles that are compatible with both the IVC rat and mouse cages (transparent polycarbonate bottles, $300 \mathrm{ml}$; Tecniplast, cat. no. ACBT0262). Our water bottles are equipped with polished AISI 316 stainless-steel bottle caps with a $25-\mathrm{mm}$ drinking nipple with a 1.8 - $\mathrm{mm}$-diameter hole for rats (Tecniplast, cat. no. ACCP2521) or a $65-\mathrm{mm}$ drinking nipple with a $2.2-\mathrm{mm}$-diameter hole for mice (Tecniplast, cat. no. ACCP6521)

- OPS objects (Equipment setup)

-Worktable on which the testing apparatus is placed (base plate, testing arena and objects). We use a normal white work table/office desk $(1,600 \mathrm{~mm} \times 800 \mathrm{~mm} \times 750 \mathrm{~mm})$ (Gispen IC)

- Viscose cleaning cloths (Sorbo, cat. no. 09660604) or other cleaning cloths (e.g., paper tissues)

- Computer (Equipment setup)

- Table to put the PC on and a chair for the experimenter to sit on. We use a normal white working table/office desk (1,600 $\mathrm{mm} \times 800 \mathrm{~mm} \times 750 \mathrm{~mm})$ (Gispen IC)

- Fluorescent red tubes and a small background light (e.g., a desk lamp, $25 \mathrm{~W}$ ) that can be dimmed with white filter paper if the light intensity is too bright (>20 lux) $\Delta$ CRITICAL Test the animals, preferably in their active phase (i.e., night phase). The OPS task does rely on visual cues. Therefore, the task should be performed in low-light conditions. The lighting conditions should be $\sim 20$ lux and equal in the different parts of the testing arena and the inside of the home cages of the animals during testing. Higher light intensities could lead to distress, behavioral disturbances and suppression of the animals' circadian rhythm ${ }^{23}$. These might affect the behavioral outcome of the animals, and hence confound the OPS results. Lighting conditions during the dark phase, when no testing occurs, should be $\sim 1$ lux.

- Radio to provide background noise in the housing room/vivarium $24 \mathrm{~h}$ per day. This way the animals become accustomed to noises in the housing room/vivarium

- Permanent marker (round-tip, black; Edding, part number 400-001))

- Electronic timer (e.g., stopwatch or timer)

- Squeeze bottles (Nalgene Right-To-Know Wash Bottle for Ethyl Alcohol, LDPE, $500 \mathrm{ml}$; Thermo Scientific, cat. no. 20-2425-0502)

- Laboratory gloves; use powder-free nitrile or vinyl gloves, as rodents dislike the texture and/or odor of latex gloves (purple nitrile gloves; Kimtech Science, cat. no. 90625)

- Transparent polysulfone plastic cages (for rats; Sealsafe Plus Green Line cages; Tecniplast, cat. no. GR900) (Tecniplast, cat. no. 1145T)

- Rack (accommodates 28 IVC Green Line cages for rats; Tecniplast, cat. no. GR28)

- Transparent polysulfone plastic cages (for mice; Sealsafe Next Blue Line cages (Tecniplast, cat. no. 1145T)

- Rack (accommodates 72 Blue Line cages for mice; Tecniplast, cat. no. TN72)

- Air-handling unit (Tecniplast, Smart Flow model)

- Circular testing arena (Equipment setup)

- (Optional) Digital camcorder with stand and mount to record OPS task trials from above (to do rescoring or scoring of the exploratory behavior later on)

- (Optional) Video-tracking system with adequate nose-tracking software (EthoVision XT; Noldus Information Technology, https:/www.noldus.com/animal-behavior-research/products/ethovision-xt? gclid=CjwKCAjwpIjZBRBsEiwA0TN1r_ucOZPXyA28tNaatilfF3StLk-sY-sP1F6zrPyHzgFBJnjBKlpNYB oC3bIQAvD_BwE)

- Personal computer

\section{Suitable cage enrichment for rats}

- Cardboard tubes, $90 \mathrm{~mm}$ in diameter $\times 125 \mathrm{~mm}$ long, thickness of the cardboard $=5 \mathrm{~mm}$ (play tunnels, rat; Bioservice/Datesand, cat. no. CS3B02A)

- Irradiated Aspen bricks, $100 \times 20 \times 20 \mathrm{~mm}$ (Bioservice/Datesand/Tapvei, cat. no. CS3C09)

- Carton nesting material (Sizzlenest, suitable for all species; Datesand, cat. no. CS1a09)

- Twisted paper nesting material (Harlan, Teklad, cat. no. 7979C.CS)

\section{Suitable cage enrichment for mice}

- Cardboard tubes, $75 \mathrm{~mm}$ in diameter $\times 38 \mathrm{~mm}$ long $/$ high, thickness of the cardboard $=1.25 \mathrm{~mm}$ (play tunnels, mouse; Bioservice/Datesand, cat. no. CS3B01)

- Red transparent polycarbonate hiding house with two holes/entrees, $100 \times 90 \mathrm{~mm}$ at the base and $75 \times$ $65 \mathrm{~mm}$ at the top (mouse house; Bioservice/Zoonlab, cat. no. 3084010) 
- Carton nesting material (Sizzlenest, suitable for all species; Datesand, cat. no. CS1a09)

- Additional twisted paper nesting material (Harlan, Teklad, cat. no. 7979C.CS)

\section{Reagent setup}

Rodents

Rats and mice should be maintained on a reversed 12-h light/dark cycle with lights off between 7:00 a.m. and 7:00 p.m. so the animals can be tested during the day in their active (dark) phase. Keep the rodents in a vivarium that is both temperature- $\left(22 \pm 1{ }^{\circ} \mathrm{C}\right)$ and humidity-controlled $(55 \% \pm 10 \%)$ with free access to food and water. Appropriate requirements for rodent husbandry and environment can be found in Appendix A of the European Convention for the Protection of Vertebrate Animals Used for Experimental and Other Scientific Purposes (ETS no. 123; https://www.coe.int/en/web/ conventions/full-list/-/conventions/treaty/123).

All OPS task experiments should be performed during the dark/active phase of the rodents (e.g., between 7:00 a.m. and 7:00 p.m.) and conducted at the same time on each experimental test day when testing repeatedly. The experimenter should not change his or her 'olfactory signature' (and avoid volatile odors, see also caution note for Step 4 of the Procedure) while interacting with the animals throughout the entire experiment. Moreover, the experimenter should have sufficient experience in scoring the exploratory behavior of the animals (see also Step 16 of the Procedure), and if applicable, should always be blinded to the experimental condition of the animals, both during testing/scoring and during the analyses of the results.

\section{Equipment setup}

\section{Rodent home cages and test rooms in the vivarium}

A sufficient number of home cages must be available for proper housing of the rodents (including bedding, food, water and cage enrichment). In our animal facility we use an individually ventilated cage (IVC) system. Conventional rodent cages will also suffice, as long as they meet the international guidelines for the proper care and use of laboratory animals. For our IVC system for rats, we use transparent polysulfone plastic (Sealsafe Plus) Green Line cages with a floor area of $904 \mathrm{~cm}^{2}$, with a standard stainless-steel wire food lid and a standard polysulfone plastic top that can hold two water bottles. For mice, we use Sealsafe Next Blue Line cages with a floor area of $435 \mathrm{~cm}^{2}$, with a standard stainless-steel wire food lid and a standard polysulfone plastic top that can hold one water bottle. Place the cages in wheel racks. We use stainless-steel racks on wheels, for holding the IVC cages. Our rack configuration accommodates 28 IVC Green Line cages for rats ( 28 cages, 4 (width) $\times 7$ (height), single sided), or 72 IVC Blue Line Next cages for mice $(72$ cages, 8 (width) $\times 9$ (height), single sided). All IVC racks are coupled to an air-handling unit. House the rodents in a dedicated room in the vivarium. The rodents can be housed in the room in which testing will take place, or in a separate room dedicated to housing only (see Step 3 of the Procedure). The home cages should contain adequate cage enrichment. Examples of suitable items for rats include cardboard tubes/play tunnels, irradiated Aspen bricks, carton nesting material and additional twisted paper nesting material). Suitable cage enrichment for mice includes cardboard tubes/play tunnels, red transparent polycarbonate hiding houses with two holes/entrees, carton nesting material, and additional twisted paper nesting material.

\section{Circular testing arena}

An arena with walls that are $400 \mathrm{~mm}$ high is required. Half of the $400-\mathrm{mm}$-high wall should be composed of gray (RAL7035) or black (RAL9005) polyvinyl chloride; the other half should be composed of transparent polyvinyl chloride. For rats, the diameter should be $830 \mathrm{~mm}$ and for mice the diameter should be $400 \mathrm{~mm}$. Our arena was made by the Maastricht University Instrument Development Engineering \& Evaluation Department (IDEE); see also Fig. 1. $\triangle$ CRITICAL In our experiments, we always use a circular testing arena to prevent a rodent from spending too much time in the corners (as is often the case when using square testing areas). Especially when placing objects in a square arena, the tendency to hide between the corners and objects intensifies. Always habituate the animals extensively to the testing arena and procedures before actual testing (see also Steps 5-9 of the Procedure). $\triangle$ CRITICAL One half of the circular testing arena must be made out of transparent material in order for the observer to perform live visual scoring (if live visual scoring is not performed, the testing arena can be entirely made out of gray or black material, and trials are subsequently filmed from above; see also Step 16 of the Procedure). 
Arena floor or base plate

A square $1,000-\mathrm{mm} \times 1,000-\mathrm{mm}$ (rats) or $600-\mathrm{mm} \times 600-\mathrm{mm}$ (mice) floor is required and should be made from either gray (RAL7035) or black (RAL9005) polyvinyl chloride. Using a permanent marker, indicate all possible locations for objects in the arena (Fig. 1), starting with a mark in the middle on the left-hand side and then making four further marks toward the front and four toward the back so that they are $60 \mathrm{~mm}$ apart for rats and $45 \mathrm{~mm}$ apart for mice Then repeat the process for the right-hand side. When the marks fade, redraw them with a permanent marker. (Our arena was made by the Maastricht University IDEE; see also Fig. 1.) $\triangle$ CRITICAL The advantage of using a gray-colored base plate is that animals with either white or dark coat colors show sufficient contrast to the floor for clear video recording and subsequently for reliable video scoring or automated tracking.

\section{Cleaning of the testing arena, base plate and objects}

The testing arena, base plate and objects must be cleaned and dried thoroughly before testing. Cleaning can be performed with a $70 \%$ (vol/vol) ethanol solution and a viscose cleaning cloth. It is important to clean the base plate and the objects after each animal/trial (see critical step note for Step 8 of the Procedure).

\section{OPS objects}

Two different sets of objects are required. Those we use for rats and mice are similar in shape and material and only differ in size. We use two different kinds of objects. These are a massive metal cube (rats: $100 \mathrm{~mm}$ high $\times 50 \mathrm{~mm}$ wide $\times 75 \mathrm{~mm}$ long; mice: $75 \mathrm{~mm}$ high $\times 25 \mathrm{~mm}$ wide $\times 50 \mathrm{~mm}$ long) with two holes in it (diameter: $19 \mathrm{~mm}$ and $15 \mathrm{~mm}$ for rats and mice, respectively), and a massive aluminum cube with a square base and a tapering top (rats: $120 \mathrm{~mm}$ high $\times 60 \mathrm{~mm} \times 60 \mathrm{~mm}$ (base); mice: $85 \mathrm{~mm}$ high $\times 45 \mathrm{~mm} \times 45 \mathrm{~mm}$ (base)) with a milled slot with a small hole (diameter: 5 $\mathrm{mm}$ ) drilled halfway through (all objects are made by the Maastricht University IDEE) (Fig. 1). $\triangle$ CRITICAL It is best to use objects that have a square/rectangular base in order to properly control the (dis)placement of the objects in the testing arena.

\section{Personal computer}

A personal computer is required to manually live-score the exploratory behavior of the rodents and/ or for video-recording of the OPS trials. We use our own in-house-developed software (ORT scoring v2.1, developed by M. Schaap (Maastricht University, 2008) as a Delphi application, using Object Pascal programming). In this program, exploration of either object is indicated by the experimenter with simple key presses, and its basic output is the total amount of time (in milliseconds) an animal explores the left and right object individually. Other software tools or timers/stopwatches could be used as well. $\triangle$ CRITICAL In our experiments, we prefer live visual scoring (and subsequent rescoring of videotaped trials by another observer) over automated scoring/tracking programs. As exploratory behavior of rodents is a rather complex behavior to be detected by automated tracking software, many false positives tend to occur with automated scoring ${ }^{34}$ (see also Step 16 of the Procedure).

\section{(Optional) Video-tracking system}

Install a digital camcorder above the testing arena on a stand and mount it in such a way that the entire floor within the testing arena is visible and the exploration behavior of the animals can be recorded from above. If using nose-tracking software, connect the camera to the video-tracking system being used. Ensure all the tracking parameters of your video-tracking system are optimized to perform proper nose-tracking of your rodents within your vivarium environment and for your OPS apparatus (testing arena, base plate and objects).

\section{Procedure}

\section{Transportation and acclimatization Timing $7 \mathrm{~d}$}

$\triangle$ CRITICAL Group housing for a week of acclimatization is required if animals have arrived from a commercial registered breeder or have been obtained from another animal facility and they have been group-housed previously in transportation boxes.

1 Group-house mice and/or rats in home cages (groups of two to three per cage) and place them on a reversed day-night cycle. Allow the animals to become acclimatized to their new cage and 
environment for $7 \mathrm{~d}$. During these $7 \mathrm{~d}$, the animals should be left undisturbed. Water and food should be available ad libitum.

$\triangle$ CRITICAL STEP Transportation of the animals and housing them in a new cage and environment is a source of stress $^{23}$ that in turn may affect the learning abilities and behavioral outcomes of rodents. This can bias your experimental results. Acclimatization and habituation to the new environment is necessary to attenuate this stress.

\section{Individual housing, housing conditions and acclimatization Timing $7 \mathrm{~d}$}

2 After acclimatization to the new environment, day-night cycle and cages, transfer the animals to individual housing with ad libitum access to food and water and allow them to become acclimatized to their new cage and individual housing for $7 \mathrm{~d}$. For easier transportation, all the animals' home cages should ideally be placed in/on movable wheel/rolling racks in case the animals need to be transported to the testing room (Step 3). Upon individual housing, number the animals on the ID tags or cages with a pen or a marker for easier identification. If the animals must be housed in a different room from where the OPS task is to be performed, they will also need to be habituated to the test room and transportation, so proceed to the next step. Otherwise, proceed to Step 4 .

$\triangle$ CRITICAL STEP Individual housing throughout the OPS procedure is preferred to ensure that the animals do not have too much interference between the learning and the test trial (Steps 10-24). Ensure sufficient cage enrichment is provided and keep this enrichment constant over the entire OPS procedure to avoid other possible interfering factors during the inter-trial intervals.

$\triangle$ CRITICAL STEP Ideally, the animals should be housed in a room that is dedicated to OPS testing. If this is possible, the animal cages should be placed on the right (50\% of animals) and left (50\% of animals) sides of the testing arena as far away from the testing arena as possible. It is always wise to check for side preferences to assess whether everything in your behavioral room is placed correctly (Anticipated results). Ensure that the spatial arrangement of the base plate, testing arena, OPS objects and all other things in the room (e.g., desk, PC) remain in similar locations throughout the OPS task. Store the OPS objects that are not used during the learning/test trial out of sight of the animals in the testing arena (e.g., under the table on which the testing arena is placed or behind the testing arena on the side of the gray/black half of the arena wall).

3 (Optional) If the animals cannot be housed in the room in which OPS testing will take place, habituate the animals to the transportation procedure and the test room on two separate days by moving them to the test room and leaving them undisturbed in the test room for at least $30 \mathrm{~min}$. The test room should be set up as if testing is to take place, and the animal cages should be placed on the right (50\% of animals) and left (50\% of animals) sides of the testing arena at a sufficient distance (as far away from the testing arena as possible).

$\triangle$ CRITICAL STEP Moving between rooms may cause stress the first few times the animals are moved.

? TROUBLESHOOTING

Habituation to the experimenter Timing $\mathbf{5}$ d; 5 min of habituation per rodent per day

! CAUTION Experimenters should keep everything consistent over the entire OPS procedure. As rodents have a highly developed olfactory system, they rely heavily upon smell. Therefore, it is important for the experimenter to keep his/her smell as similar as possible. Keep a personal lab coat within the testing room that is not worn by someone else. Furthermore, this also implies that the experimenter must use the same deodorant, shampoo, shower gel, hair gel and so on throughout the entire OPS procedure. Avoid the use of volatile odors or perfume. Changing the 'olfactory signature' of the experimenter is a cause of stress that will lead to inconsistent results.

! CAUTION If using gloves when handling animals, always use powder-free nitrile or vinyl gloves, as rodents dislike (the texture and/or odor of) latex gloves.

$\triangle$ CRITICAL Animals must become habituated to the experimenter.

4 Habituate the animals to the experimenter. If the planned experiment requires the testing of naive animals or animals in which restraining procedures and/or injections are not required (e.g., transfected animals, animals with a minipump), follow option A at least twice for 5 min on separate days (days 1 and 3), leaving $1 \mathrm{~d}$ (day 2) between, during which the animals are left undisturbed. If the planned experiment does require restraining and/or injection of the animals, follow option $\mathrm{B}$ (see also the flowchart in Fig. 3). 
(A) Habituation in the absence of additional procedures

(i) Gently open the lid of the home cage and put your hand into the cage; let the rodent smell and explore your hand for a short period of time. The animals should be allowed sufficient time to become aware of your presence before moving to the next step.

(ii) Pick up the rodent gently, and after lifting it out of its cage, place it on the arm of your lab coat. Pick up a rat by the thorax, if possible. If the rat keeps fleeing or is behaving unpredictably, pick it up by the base of the tail. Pick up mice by the base of the tail. During the first handling sessions, rats will typically move toward your cubital fossa (elbow pit) and tend to hide their head there. Just let the rat do this for a while and gently pet the animal so that it becomes accustomed to being touched/approached from above. Mice tend to become less accustomed to handling than rats. Another, less stressful option for mice is to let the mouse climb onto an object (e.g., a small house or a cup), after which it can be placed on your arm.

(iii) Leave the rodent to explore or rest on your arm for $5 \mathrm{~min}$; after this, gently return it to the home cage. During later sessions, if using a rat, consider picking it up and putting it back in a different position on your arm a couple of times, to allow the animal to become accustomed to being handled.

$\triangle$ CRITICAL STEP If rodents are not properly habituated to the handling procedures, they will not be comfortable in the testing apparatus, which can bias experimental results. We advise handling the animals at least twice on separate days, but if the animals do not seem comfortable after $2 \mathrm{~d}$, this step can be repeated until the animals appear calm and comfortable. ? TROUBLESHOOTING

(B) Habituation when additional procedures will be carried out

$\triangle$ CRITICAL If the planned experiment involves drug testing that requires administration of injections, it is important to habituate the animals to this procedure as well as to handling. Habituation sessions should be performed for both the restraining of an animal and the actual injection(s). Restraining and injecting an animal for the first time right before the actual test leads to excessive stress, which can bias the experiment. We recommend giving the animals at least one saline injection during habituation, using the route of administration planned for the experiment. If repeated injections are necessary, consider alternatives, such as the use of minipumps, to reduce the discomfort of the animals.

(i) Habituate animals to the experimenter first by carrying out Step 4A at least twice for $5 \mathrm{~min}$ on separate days (days 1 and 3; leave $1 \mathrm{~d}$ between, during which the animals are left undisturbed) (Step 4A, Fig. 3).

(ii) On the subsequent day (day 4), handle the animals as described in option A and also restrain the animal once for a few seconds during the 5-min habituation without injecting it. Gently pet or leave the animal on your arm for the rest of this habituation phase. To keep stress levels as low as possible, restrain the animal smoothly and without hesitation.

(iii) On the following day (day 5), again remove the animal from its cage, restrain it as described in the previous step and administer a saline injection via the route that will be used during the OPS task (e.g., i.p., p.o., s.c. or i.m.).

$\triangle$ CRITICAL STEP If multiple injection routes are required, carry out only one injection.

(iv) If multiple injection routes will be required, leave the animals undisturbed for $1 \mathrm{~d}$ (day 6) and then repeat the previous step on day 7 , administering a saline injection via the second required route of administration.

\section{Habituation to the OPS apparatus Timing typically, $5 \mathrm{~d}$}

$\triangle$ CRITICAL After the rodents are habituated to the handling by the experimenter, they must be habituated to the testing apparatus.

$\Delta$ CRITICAL The testing arena and the objects must be cleaned thoroughly after each animal with a cleaning cloth and a $70 \%$ (vol/vol) ethanol solution in order to remove all olfactory traces that could attract or repel an animal toward or away from an object or location. Make sure that the ethanol has completely evaporated when the next animal is introduced into the testing arena (not doing this will influence the results of the experiment).

5 Handle the animal for $5 \mathrm{~min}$, as described in Step 4A. If using mice, in order to increase their arousal at testing, next place the mouse in an empty cage (only bedding, no enrichment) for 4 min before moving to the next step. If using rats instead, proceed to the next step.

6 Place the animal in an empty testing arena (facing the middle of the transparent segment of the arena or facing the middle of the gray/black half of the arena). Let the animal freely explore the arena 
for $5 \mathrm{~min}$ (during this time the second animal can be handled as described in Step 5). After 5 min, gently remove the animal from the arena and place it back in the home cage and the holding rack.

7 Repeat Steps 5 and 6 on the next day (day 2 of OPS habituation).

8 On the next day (day 3 of OPS habituation), place the first set of similar objects in the testing arena randomly at the indicated possible locations. Make sure one object is located on the left vertical axis and the other one is located on the right vertical axis (e.g., Fig. 2). Repeat Steps 5 and 6 to provide the animals the opportunity to become familiarized with the objects. Let the animal freely explore the testing arena with both objects for $5 \mathrm{~min}$.

$\triangle$ CRITICAL STEP For new experimenters, it helps to practice scoring during the habituation phase. This can be done by carrying out Step 16A while the animal is exploring the testing arena.

9 Leave the animals to rest for $1 \mathrm{~d}$ (day 4 of OPS habituation) and on the following day (day 5 of OPS habituation), repeat Step 8, but this time use the second set of similar objects and different locations along the left and right vertical axes.

\section{OPS task procedure and training-1-h inter-trial interval $\bigcirc$ Timing $6 \mathrm{~d} ; 2 \mathrm{~h}$ per day for a cohort of 12 rats or ten mice}

! CAUTION OPS testing should always be performed in a quiet room and area. Even soft noises can distract the animals and influence and/or bias the results.

$\triangle$ CRITICAL The following steps should be carried out carefully in order to obtain reliable task performance from the animals. The procedures for T1 (Steps 10-20) and T2 (Steps 21-24) are similar, except that in T2 one of the two similar objects is displaced along a vertical axis. Placement should be determined by a randomized scheme (Tables 1 and 2) and be to one of five different positions along a vertical axis (ranging from the same position as in T1, to a maximal displacement similar to the new location in the OLT ${ }^{15}$; see also Fig. 3 and Box 1).

10 OPS task procedure-learning trial (T1). Thoroughly clean the testing arena, base plate and OPS objects with a cleaning cloth and a $70 \%$ ( $\mathrm{vol} / \mathrm{vol})$ ethanol solution. When testing mice, prepare an empty cage with only bedding and put this cage somewhere in the room, away from the testing arena.

$\triangle$ CRITICAL STEP Ensure that the testing arena is on the base plate at the same position at which it was previously, as it must be in the same place throughout the entire experiment. Make sure that all possible object positions in the testing arena are clearly visible (Fig. 1).

$\triangle$ CRITICAL STEP Store the OPS objects that are not used during the trial out of sight of the animals in the testing arena (e.g., under the table the testing arena is on or behind the testing arena behind the gray- or black-colored half of the arena wall).

11 Place two similar objects in the testing arena at the starting positions. The starting positions are two horizontally symmetrical locations at the middle line of the testing arena $\sim 25 \mathrm{~cm}$ (rat arena) or $\sim 12$ $\mathrm{cm}$ (mouse arena) from the wall on each side (position 1, Fig. 2 and Tables 1 and 2).

$\triangle$ CRITICAL STEP The experimenter must choose with which object set (1 or 2, Fig. 1) to start. It is important to alternate between the two object sets on subsequent testing days (e.g., rat 1 gets to explore object set 1 on test day 1 , object set 2 on test day 2, object set 1 again on test day 3 and so on). A randomization scheme should be designed in which both the positions to be tested in T2 (Steps 21-24) and the objects are used in a balanced manner to prevent potential biases due to preferences for particular locations or objects (Table 1).

$\triangle$ CRITICAL STEP Although they are similar, allocate each individual object to one side of the testing arena and keep there for this entire test day. Small differences in the texture of the objects might be more salient to rodents than they are to us and might subsequently lead to inconsistent performance of the animals. In addition, make sure that the objects are always oriented the same way (i.e., holes in the objects should always face the same direction).

12 When using mice, place the mouse in an empty cage (only bedding, no enrichment) for $4 \mathrm{~min}$ immediately preceding $\mathrm{T} 1$ to increase the arousal of the mouse at testing.

13 Start the timer or stopwatch to time the total time required to carry out T1 for the animal (5 min per rat; 6 min per mouse).

$\triangle$ CRITICAL STEP Tracking the entire time with each animal is necessary to precisely time the appropriate inter-trial interval $(1 \mathrm{~h})$ for each individual animal.

14 Gently take the home cage of the animal out of the rack and place it on a table in the room.

$\triangle$ CRITICAL STEP Do not place the home cage of the animal in close proximity to the testing arena, as this could potentially attract the animal toward its cage while it is in the OPS task. 
In our laboratory, we place the home cage of the animal on the same table where the PC is located.

15 Take the animal out of its home cage and place it in the testing arena containing the two symmetrically placed objects facing the middle of the front transparent half of the arena or facing the middle of the gray/black half of the arena (i.e., facing away from the objects).

$\triangle$ CRITICAL STEP Ensure the starting point and the direction the animals face when placed are kept identical during the entire OPS habituation, training and experimental procedures. Changing the starting position can lead to biased results. As this is a spatial task, the animals will always be placed closer to one object than the other during the test trial (T2, Steps 21-24). As long as the positions of the objects and the experimental conditions are tested in a balanced manner, the possible confounding effect of animals being placed in closer proximity to one object, as opposed to the other, is minimized. $\triangle$ CRITICAL STEP Make sure that the cleaning ethanol has completely evaporated when the animal is introduced into the testing arena (not doing this can influence the results of the experiment).

16 Start a second timer or stopwatch to precisely time the period in the arena ( $3 \mathrm{~min}$ per rat; $4 \mathrm{~min}$ per mouse, see Tables 4 and 5) and ensure the correct inter-trial interval (1 h) for each individual animal. In addition, start scoring and/or recording the behavior of the animals. For live visual scoring of the exploratory behavior of the rodents, follow option A. Video-recording of the trial is also advisable, if possible, as described in option B to (manually or automatically) score or enable rescoring of the exploratory behavior. Option $\mathrm{C}$ describes how to score exploratory behavior automatically using a video-tracking system with nose-tracking software. Option B offers some advantages; for instance, because observation is from above, it is always possible to see what an animal is doing and the animal does not become hidden behind an object, as can happen during live scoring. In addition, the trials can be scored by different observers at different moments, which allows for cross-comparisons (and measurement of inter-observer reliability), and a permanent archive can be created, which allows later access for rescoring or meta-analyses. An example of an OPS experimental trial is shown in the Supplementary Information. Videos of T1 and T2 for the same mice are shown in Supplementary Videos 1 and 2, respectively. The scoring of the exploration and discrimination performance of this mouse and calculations of the parameters outlined in Table 3 are shown in the Supplementary Data. $\triangle$ CRITICAL STEP Total exploration times should always be minimally $7 \mathrm{~s}$ in T1 for familiarized animals to properly learn the spatial arrangement of the objects in the testing arena ${ }^{30}$. Total exploration times should always be minimally $10 \mathrm{~s}$ in T2 for familiarized animals in order to draw reliable conclusions with regard to the discrimination performance ${ }^{30}$.

(A) Live visual scoring

(i) Carefully monitor the animals while in the testing arena. The experimenter should be placed at a position within the room where he/she can see as much of the testing arena as possible and should look through the transparent part of the testing arena in order to monitor the animal. A disadvantage of this way of scoring behavior is that the experimenter cannot see what an animal is doing while it is behind one of the objects, exploring the lower part of the object. Only exploratory behavior that can properly be seen should be scored. Score the cumulative exploration behavior of the animals with appropriate software or stopwatches/ timers. The observer should remain as quiet as possible while the animal is interacting with the objects. Even soft sudden noises (e.g., cracking of a chair) could distract the animals and influence the exploratory behavior. Exploration is defined as follows: directing the nose to the object at a distance of no more than $2 \mathrm{~cm}$ and/or touching the object with the nose. Sitting on or leaning against an object is not considered to be exploratory behavior; the animals should only be scored as exploring when actively investigating an object. We recommend using a personal computer to manually score (live visual scoring) the exploratory behavior of the rodents. Alternatively, separate stopwatches can be used to measure the amount of exploratory behavior per object, but this is more time-consuming and demanding than using simple software that accumulates exploration time.

$\triangle$ CRITICAL STEP Well-trained experimenters are required; new experimenters should have already practiced scoring during Steps 8 and 9, if necessary.

(B) Video-recording for scoring

(i) Record the animals' behavior using a pre-installed digital camcorder.

(C) Automated scoring by video-tracking system

(i) Video-track the OPS trials for object exploration. The regions of interest that the software must score are the areas around the objects (no more than $2 \mathrm{~cm}$ away from the object, see Step 16A) and the objects themselves. The software should continuously track the nose of 
the animal and only score behavior as exploratory if the nose of the animal is within the indicated region of interest. An advantage of automated scoring by a video-tracking system is that the total distance moved as a measure for locomotor behavior of the animals can also be analyzed. To accomplish this, the center point of the animal can be tracked (so nose tracking is not necessary). This way, the potential confounding effect of locomotor deficiencies of the animals can be assessed.

$\triangle$ CRITICAL STEP Before analyzing the OPS dataset generated by the video-tracking system, check whether manual scoring from a few video files correlates to the data generated by the video-tracking system. All parameters must be calibrated in order to obtain the most reliable results.

? TROUBLESHOOTING

17 After the trial duration expires (3 or $4 \mathrm{~min}$ ), take the animal out of the arena and place it back in the home cage and the holding rack to be kept ready for its learning trial (T2), which must start in $1 \mathrm{~h}$. Store the acquired data.

18 Clean the testing arena, base plate and OPS objects with a cleaning cloth and a 70\% (vol/vol) ethanol solution. Again, store the cleaned objects out of sight of the animals.

$\triangle$ CRITICAL STEP It is recommended to clean the used objects after each animal instead of cleaning the to-be-used objects before each animal. This ensures that all the ethanol will have evaporated from the arena and objects before using them again.

$\triangle$ CRITICAL STEP Steps 11-18 must be completed within $5 \mathrm{~min}$ for a rat or $6 \mathrm{~min}$ for a mouse to keep on schedule for time-planning.

19 Repeat Steps 11-18, using the objects and position determined by the randomization schedule on each animal until the remaining 11 rats (12 rats in total) or nine mice (ten mice in total) have been through T1.

\section{? TROUBLESHOOTING}

20 When an hour has elapsed since the first animal completed testing in T1, proceed to the next step for the second trial (T2) (Steps 21-24). If using 12 rats or ten mice, with 5 min or 6 min having been used for each rat or mouse trial, $1 \mathrm{~h}$ will have elapsed since the first animal completed testing in T1 ((12 rats $\times 5 \mathrm{~min})$ or (ten mice $\times 6 \mathrm{~min}))$, so you should immediately proceed to the next step.

21 OPS task procedure-test trial (T2). Repeat Steps 11-18 with the same set of objects that was used for the animal in $\mathrm{T} 1$ but with one of the two objects displaced to one of five positions along the vertical axis indicated on the base plate of the OPS apparatus. The position at which to place the object should be determined by the randomization scheme (Tables 1 and 2).

$\triangle$ CRITICAL STEP Ensure that the object that was presented on the left side of the testing arena in $\mathrm{T} 1$ is again on the left side in T2 and that the object presented on the right side in T1 is now again on the right side of the testing arena in T2.

$\triangle$ CRITICAL STEP Use a balanced randomization scheme for both the objects and the object positions in T2 to prevent potential biases due to preferences for particular locations or objects. An example is given for 12 animals in the appended randomization scheme, in which positions 1,3 , and 5 , or only position 3, are tested in a balanced way with two objects (Tables 1 and 2, respectively). ? TROUBLESHOOTING

22 Repeat Step 21 for the remaining 11 rats (12 rats in total) or nine mice (ten mice in total)tested in T1, with objects used and placed as determined by the predetermined randomization scheme. ? TROUBLESHOOTING

23 If $5 \mathrm{~min}$ in total is used for rats and $6 \mathrm{~min}$ in total used for mice, $2 \mathrm{~h}$ will have elapsed since the first animal was tested in $\mathrm{T} 1$ ( $(12$ rats $\times 5 \mathrm{~min} \times$ two trials $)$ or (ten mice $\times 6 \mathrm{~min} \times$ two trials $))$. If desired, repeat Steps $10-22$ with a second cohort of 12 rats or ten mice.

? TROUBLESHOOTING

24 After finishing the OPS experiment, score any videotaped material that has not been automatically analyzed by software as described in Step 16A and analyze the stored data using spreadsheet software or a statistical program (Anticipated results).

? TROUBLESHOOTING

\section{Further OPS training Timing $6 \mathrm{~d}$}

$\triangle$ CRITICAL After the rodents are habituated to the experimenter, the testing arena, the OPS objects and the OPS task, the actual training in the OPS task can start. This measures the performance of the animals in the task and assesses whether they show reliable pattern separation performance. The most 
straightforward way of measuring pattern separation performance is by first establishing performance at positions 1, 3 and 5 in the test trial (T2, Steps 10-24, see also Box 1). When the linear relationship

(Fig. 4) is established, the actual OPS experiment (Steps 32-36) can start. The total exploration times should always be minimally $7 \mathrm{~s}$ in $\mathrm{T} 1$ and $10 \mathrm{~s}$ in $\mathrm{T} 2$, in order to draw reliable conclusions with regard to the discrimination performance of familiarized animals ${ }^{30}$.

25 Leave the animals for a day before further testing.

26 On the next day, repeat Steps 10-24, using the same objects for T1 and T2 but displacing the object in the T2 to position 5 (where the displacement of the object is maximal). The expected outcome is that the $\mathrm{d} 2$ value will be $\sim 0.30-0.45$ (see Anticipated results and Fig. 2).

$\triangle$ CRITICAL STEP Performance at position 5 is most informative in this first stage of training because it shows whether the animals have the capacity to learn. Displacement to position 5 enables the highest performance to be achieved ( $\mathrm{d} 2$ of $\sim 0.30-0.45$ ). Starting with other positions will be less informative at this stage (e.g., starting with position 1, where a $\mathrm{d} 2$ of $\sim 0.00$ is anticipated, will not be informative with regard to the learning abilities of the rodents, as there is no novelty for the animals to explore and hence no way of assessing whether the animals are able to learn spatial information properly). A low-discrimination performance at position 5 indicates deviant behavior that might relate to anxious or stressed behavior or erroneous displacement of the object(s) in T2. ? TROUBLESHOOTING

27 Leave the animals for a day before further testing.

28 On the next day, repeat Steps 10-24, starting with the second set of similar objects for T1 and T2 but displacing the object in T2 to position 3 (where the displacement of the object in T2 is halfway between the starting position, position 1, and the maximum position, position 5). The expected outcome is that the $\mathrm{d} 2$ value will be $\sim 0.15$ (Anticipated results and Fig. 2).

? TROUBLESHOOTING

29 Leave the animals for a day before further testing.

30 On the next day, repeat Steps 10-24, starting with the first set of similar objects for T1 and T2 and not displacing the object in T2 but leaving it at position 1 . The expected outcome is that the $\mathrm{d} 2$ value will be $\sim 0.00$ (Anticipated results and Fig. 2).

? TROUBLESHOOTING

31 Leave the animals for a day before further testing.

PAUSE POINT This rest period can also extend over the weekend, if desired.

\section{OPS experiment Timing $5 \mathrm{~d}, \mathbf{2} \mathrm{h}$ per day for a cohort of 12 rats or ten mice}

$\triangle$ CRITICAL After the rodents have been habituated and trained for the OPS procedure and show reliable OPS performance (Figs. 2 and 4), the actual experiment can start. We provide an example below for an experimental week ( 3 testing days) during which three OPS experiments are performed in which different experimental conditions are tested at OPS position 3 in order to assess whether the experimental conditions impair or improve OPS performance.

$\triangle$ CRITICAL When the testing involves the administration of drugs, timing of the testing should be customized according to the $T_{\max }$ of the drug. Examples for a drug with a $T_{\max }$ of 30 min that must be maximally active during the memory-acquisition phase is shown in Tables 4 (mice) and 5 (rats).

$\triangle$ CRITICAL During drug testing, the half-life of the drug should always be taken into account in order to prevent accumulation of the drug in the system. In general, an interval of at least six times the half-life of a drug (98.4\% of the drug eliminated) should be interposed between subsequent drug testing/experiments. $\triangle$ CRITICAL When testing different experimental groups, experimenters should always be blinded to the treatments or conditions of the animals.

$\triangle$ CRITICAL A randomization scheme should be designed in which the objects, the locations to be tested in T2 (i.e., left and right) and the experimental conditions are used in a balanced manner to prevent confounding of the results (Table 2).

32 Monday. Repeat Steps 10-24, starting with the first set of familiar objects and placing the objects at position 3 during T1. In T2 displace the object to position 3. The expected outcome is that the $\mathrm{d} 2$ value will be $\sim 0.15$ in the control or vehicle condition (Anticipated results and Fig. 2). Experimental conditions that improve or impair pattern separation performance will show an increase (e.g., a $\mathrm{d} 2$ value of 0.30 ) or a decrease (e.g., a d2 value of 0.00 ) in the discrimination index (d2), respectively. ? TROUBLESHOOTING

33 Tuesday. Leave the animals for a day with no testing. 
34 Wednesday. Repeat Step 32 but use the second set of similar objects. ? TROUBLESHOOTING

35 Thursday. Leave the animals for a day with no testing.

36 Friday. Repeat Step 32. ? TROUBLESHOOTING

\section{Ongoing experiments Timing Variable}

37 Perform further experiments. Animals can be tested repeatedly in the OPS task, so, if desired, further studies can be undertaken with the same sets of objects. To prevent recent memory effects of the objects, at least $48 \mathrm{~h}$ should be interposed between subsequent experiments. This means that during 1 week (Monday-Friday), three OPS experiments can be performed in one cohort of animals (Monday, Wednesday and Friday) (Fig. 3).

\section{Additional endpoints Timing Variable}

38 After the necessary experimental conditions are tested in the OPS experiment and if required by the experimental design, anesthetize the animals, then euthanize and dissect them for tissue collection according to the requirements of your experiment and national and institutional regulations. In our laboratory, anesthesia and euthanasia are performed using methods that conform to Annex IV of Directive 2010/63/EU. The specific method used is dependent on the exact studies undertaken and, for example, whether specific tissue is retained for downstream analysis.

! CAUTION All personnel involved in anesthesia or euthanasia of animals should have all the required licenses and be adequately trained to perform these procedures.

\section{Troubleshooting}

Troubleshooting advice can be found in Table 6 .

\section{Table 6 | Troubleshooting table}

\begin{tabular}{|c|c|c|c|}
\hline Step & Problem & Possible reason & Solution \\
\hline \multirow[t]{4}{*}{$\begin{array}{l}3,26,28, \\
30,32,34 \\
\text { and } 36\end{array}$} & $\begin{array}{l}\text { The rodent shows side preference } \\
\text { in the testing arena }\end{array}$ & $\begin{array}{l}\text { Home cages are located on the same side of } \\
\text { the testing arena that the animal prefers }\end{array}$ & $\begin{array}{l}\text { House (or place) the animals on both sides (left and } \\
\text { right OR front and back) of the testing arena }\end{array}$ \\
\hline & & Stressors or noises in the testing room & $\begin{array}{l}\text { Check the testing room for possible stressors (e.g., } \\
\text { temperature, humidity, lighting conditions, noises and } \\
\text { airflow) }\end{array}$ \\
\hline & & The testing arena is unequally lit & $\begin{array}{l}\text { Make sure the testing apparatus is equally lit across } \\
\text { the base plate }(\sim 20 \text { lux })\end{array}$ \\
\hline & & $\begin{array}{l}\text { Home cage of the animal is too close } \\
\text { to one side of the testing arena }\end{array}$ & $\begin{array}{l}\text { Place the home cage further away from the testing } \\
\text { arena }\end{array}$ \\
\hline \multirow[t]{3}{*}{ 4A(iii) } & $\begin{array}{l}\text { After handling the rodent twice for } \\
5 \text { min, the animal still appears } \\
\text { uncomfortable }\end{array}$ & The animal is stressed or anxious & $\begin{array}{l}\text { More handling is necessary before starting the actual } \\
\text { OPS procedure. Handle this animal twice more for } \\
5 \text { min each on separate days (Step 4) }\end{array}$ \\
\hline & & & Check the testing room for possible stressors \\
\hline & & & $\begin{array}{l}\text { When transporting animals to a testing room, prolong } \\
\text { the time to acclimatize before the start of the } \\
\text { experiment }\end{array}$ \\
\hline \multirow[t]{2}{*}{16} & $\begin{array}{l}\text { Automated nose-tracking with } \\
\text { a video-tracking system and } \\
\text { software leads to false-positive } \\
\text { object exploration }\end{array}$ & $\begin{array}{l}\text { Unreliable/inconsistent nose-tracking of the } \\
\text { animal-e.g., when an animal is sitting on top } \\
\text { of an object and is looking up, its nose will be } \\
\text { in the indicated region of interest. Although }\end{array}$ & $\begin{array}{l}\text { Filming from multiple sides of the testing arena might } \\
\text { solve this issue. The video-tracking system and } \\
\text { software must be compatible with this. }\end{array}$ \\
\hline & & $\begin{array}{l}\text { this is not considered to be exploratory } \\
\text { behavior, a video-tracking system might not } \\
\text { be able to tell the difference }\end{array}$ & $\begin{array}{l}\text { Consider manually scoring the trials from recorded } \\
\text { video files }\end{array}$ \\
\hline
\end{tabular}




\section{Table 6 (continued)}

Step Problem

$22,24,26$, the objects sufficiently

$28,32,34$, (i.e., $<7$ s during $\mathrm{T} 1$ and/or

and $36<10$ s during T2)
16, 19, 21, The rodent does not explore

\section{Possible reason}

The animal's arousal is too low during testing

The animal is stressed or anxious

The animal is not interested in the objects

When drugs have been administrated to the animal, arousal and/or locomotor activity of the animal may be altered (side-effects)

$21,22,23, \mathrm{~d} 2$ values at positions 1,3 and $24,26,28, \quad 5$ are not similar to those of $30,32,34$, the examples given in Fig. 4 and 36
Objects are still too novel (leads to higher performance than anticipated)

The animal is stressed or anxious

The $\mathrm{d} 2$ value does significantly differ from zero/chance level while using position 1 and/or 2 in $\mathrm{T} 2(P<0.05)$

Side preference in the testing arena
The baseline (i.e., no experimental condition) $\mathrm{d} 2$ value does not significantly differ from zero/ chance level $(P>0.05)$ while using position 3,4 or 5 in T2 (i.e., OPS performance is too low)

The baseline (i.e., no experimental condition) $\mathrm{d} 2$ value does not significantly differ $(P>0.05)$ from that of position 1 while using position 3,4 or 5 in T2

Pattern separation performance varies within the same position between similar groups on different experimental days
Objects are incorrectly placed in the testing arena during T1, T2, or both

Animal is stressed or anxious

Objects are incorrectly placed in the testing arena during $\mathrm{T} 1, \mathrm{~T} 2$, or both

Animal is stressed or anxious

Objects are incorrectly placed in the testing arena during $\mathrm{T} 1, \mathrm{~T} 2$, or both

Testing conditions differ between experimental days

\section{Solution}

For mice: place the mouse in an empty cage (only bedding, no enrichment) for 4 min immediately preceding the learning and test trials (both $\mathrm{T} 1$ and $\mathrm{T} 2$ ) to increase the exploratory behavior of the mouse (Step 12)

Check the testing room for possible stressors (e.g., temperature, humidity, lighting conditions, noises and airflow); make sure the home cage has not been cleaned on the experimental day; when transporting animals to a testing room, prolong the time to acclimatize before the start of the experiment; increase the trial duration (e.g., by $1 \mathrm{~min}$ )

Make sure to have interesting objects for the animals to explore (such as those shown in Fig. 1). Objects can be made more attractive by adding holes to them (always habituate/familiarize the animals with the objects before testing)

If possible, administer the drug earlier; check whether the effects hold after repeated administration; increase the trial duration (e.g., by $1 \mathrm{~min}$ )

Repeat Steps 8 and 9, i.e., show both object sets again

While in the experiment (Steps 32-36), repeat Steps 25-31 until stable OPS performance is shown

Check the testing room for possible stressors (e.g., temperature, humidity, lighting conditions, noises and airflow)

Make sure the home cage has not been cleaned on the experimental day

Check which side is preferred or avoided by the animal and subsequently check the behavioral room to see whether there are factors that could explain this behavior. Known causes of side preferences are unequal light conditions on the floor of the testing arena, home cage that is too close to the testing arena, housing of conspecifics on one side of the testing arena, and uneven airflow or noises in adjacent rooms (stressors).

Exclude the animal

Carefully place the objects at the correct locations (both $\mathrm{T} 1$ and $\mathrm{T} 2$ )

Check the testing room for possible stressors (e.g., temperature, humidity, lighting conditions, noises and airflow); make sure the home cage has not been cleaned on the experimental day

Carefully place the objects on the correct locations (both $\mathrm{T} 1$ and $\mathrm{T} 2$ )

Check the testing room for possible stressors (e.g. temperature, humidity, lighting conditions, noises and airflow); make sure the home cage has not been cleaned on the experimental day

Carefully place the objects on the correct locations (both $\mathrm{T} 1$ and $\mathrm{T} 2$ )

Always start testing at the same time on an experimental day (effects of circadian rhythm ${ }^{19}$ ) 


\begin{tabular}{|c|c|c|c|}
\hline Step & Problem & Possible reason & Solution \\
\hline & \multirow{6}{*}{$\mathrm{d} 2$ index is 1 or -1} & $\begin{array}{l}\text { Animals are stressed or anxious } \\
\text { during an experimental day }\end{array}$ & $\begin{array}{l}\text { Check the testing room for possible stressors } \\
\text { (e.g., temperature, humidity, lighting conditions, } \\
\text { noises and airflow); make sure the home cage } \\
\text { has not been cleaned on the experimental day; } \\
\text { when transporting animals to a testing room, } \\
\text { prolong the time to acclimatize before the start } \\
\text { of the experiment; shared testing room-mark } \\
\text { the position of the setup and place it back in } \\
\text { precisely the same location }\end{array}$ \\
\hline & & $\begin{array}{l}\text { The animal hides behind one of the } \\
\text { objects, resulting in observed exploration } \\
\text { of only one object }\end{array}$ & $\begin{array}{l}\text { Animal is stressed; exclude the animal from the } \\
\text { dataset }\end{array}$ \\
\hline & & Side preference (lateralization) & $\begin{array}{l}\text { Resolve side preferences as indicated elsewhere in } \\
\text { this table }\end{array}$ \\
\hline & & $\begin{array}{l}\text { Objects are still too novel (leads to } \\
\text { higher performance than anticipated) }\end{array}$ & $\begin{array}{l}\text { Repeat Steps } 8 \text { and 9, i.e., show both object sets } \\
\text { again. }\end{array}$ \\
\hline & & & $\begin{array}{l}\text { While in the experiment (Steps } 32-36 \text { ), repeat Steps } \\
25-31 \text { until stable OPS performance is acquired }\end{array}$ \\
\hline & & Animal is stressed or anxious & $\begin{array}{l}\text { Check the testing room for possible stressors (e.g., } \\
\text { temperature, humidity, lighting conditions, noises and } \\
\text { airflow); make sure the home cage has not been } \\
\text { cleaned on the experimental day }\end{array}$ \\
\hline
\end{tabular}

Step 1, transportation and acclimatization: $7 \mathrm{~d}$

Steps 2 and 3, individual housing, housing conditions and acclimatization: $7 \mathrm{~d}$

Step 4, habituation to the experimenter and OPS apparatus: $5 \mathrm{~d}$

Steps 5-9, habituation to the OPS apparatus: $5 \mathrm{~d}$

Steps 10-24, OPS task procedure and training-1-h inter-trial interval: $6 \mathrm{~d}$

Steps 25-31, further OPS training: $6 \mathrm{~d}$

Steps 32-36, OPS experiment: $5 \mathrm{~d}, 2 \mathrm{~h}$ per day for a cohort of 12 rats or ten mice

Step 37, on-going experiments: variable

Step 38, additional endpoints: variable

\section{Example schedule for a typical experiment}

Step 1 (days 1-7), acclimatization of newly arrived animals

Steps 2 and 3 (days 8-14), acclimatization to individual housing

Step 4 (days 15-19), acclimatization to handling, $5 \mathrm{~d}$

Steps 5-9 (days 20-24), acclimatization to the OPS apparatus: $5 \mathrm{~d}$

Steps 10-31 (days 25-36), OPS task training, $12 \mathrm{~d}$

Steps 32-36 (days 37-41), three OPS experiments (in $5 \mathrm{~d}, \mathrm{~N}=20-24$ )

Step 37 (day 42 and beyond), ongoing experiments (variable; three OPS experiments per week)

Step 38 (day of/after last experiment), additional endpoints (variable)

\section{Anticipated results}

\section{Statistical analysis}

The OPS task provides measures for exploration time and discrimination performance. The outcome measures of the task are the times spent by rodents exploring each object location during the learning trial (T1) and the test trial (T2). The times spent in exploring the two symmetrically placed objects in $\mathrm{T} 1$ are represented by 'a1' and 'a2', respectively. The times spent in exploring the stationary and the moved objects in T2 are represented by 'a3' and 'b', respectively. From these exploration times, the following variables are calculated: e1, e2 and $\mathrm{d} 2$ (Table 3). Total exploration times in both trials should be sufficient (i.e., T1/e1 $\geq 7 \mathrm{~s}$ and T2/e2 $\geq 10 \mathrm{~s}$ for familiarized animals) in order to reliably assess discrimination performance ${ }^{30}$. If one or both of these criteria are not met, exclusion of the 
animal is warranted. The $\mathrm{d} 2$ index is a relative measure of discrimination performance, as it is corrected for total exploratory activity in the test trial (e2). The $\mathrm{d} 2$ index can range from -1 to 1 ; with -1 indicating complete preference for the familiar/stationary object location, 1 indicating complete preference for the novel object location and 0 signifying no preference for either object location (chance performance). A d2 index should never be 1 or -1 , as this means an animal completely ignored one of the objects or sides of the testing arena (see ? TROUBLESH00TING for Steps 21-24). Ignoring one side or object is deviant behavior and often emerges when an animal is anxious. Animals should always explore both objects in order to draw reliable conclusions with regard to their pattern separation performance ${ }^{15}$. Animals with a $\mathrm{d} 2$ index of -1 or 1 should be excluded from the dataset, as this performance and outcome will bias the results. Additional measures, such as total distance moved during both trials, can serve as an assessment of locomotor activity, which can be a valuable readout in, for example, pharmacological studies or when using deficit models (e.g., transgenic or pharmacological models). Total distance moved can be easily assessed when using an automated scoring program (see also Step 16C).

Spreadsheet software (e.g., Microsoft Excel) should be used to enter and organize the different OPS parameters. For faster OPS data analysis, reusable templates can be made using spreadsheet software (see also Supplementary Videos 1 and 2 and the Supplementary Data for information about scoring of video files; output and calculations of the parameters are outlined in Table 3). The OPS parameters can be analyzed using a statistical program (e.g., IBM SPSS Statistics). One-sample $t$-statistics can be performed in order to assess whether the $\mathrm{d} 2$ index significantly differs from zero (i.e., chance-level performance, $(P<0.05))$. However, comparison of the value of $\mathrm{d} 2$ with the value 0 with no variance may not be the most suitable way to analyze performance because there is an increased chance of making a type-I error. Another possibility is to perform a $t$ test that compares to a fictive group that shows no discrimination. The fictive group is a standard dataset with a mean $\mathrm{d} 2$ value of 0.00 (no discrimination, i.e., chance performance) and an s.e.m. that is usually observed in the same type of experiments (i.e., 0.07). To obtain an average s.e.m., an earlier study performed in our lab calculated the mean value of ten previous random independent ORT experiments, the s.e.m. was 0.07. On the basis of these data, a fictive group was constructed for the ORT. As the $\mathrm{d} 2$ index is also used in the OPS task, similar variance in the data can be expected ${ }^{35}$. Experimental conditions, including the different positions, should also be compared using one-way ANOVAs, repeated measures ANOVAs or pairedsamples $t$-statistics, depending on the experimental design (e.g., performance difference between different positions, groups of animals within positions, drug treatments). When the overall ANOVA is significant $(P<0.05)$, post hoc $t$ tests can be performed. Normally, an a level of 0.05 is considered significant (i.e., $P<0.05$ ). A d2 value that is significantly different from 0 (as indicated by one-sample $t$-statistics, $(P<0.05))$ signifies an intermediate effect, and as such, already indicates recognition of the familiar object location, implying functioning pattern separation. A significant difference from a fictive group $(P<0.05)$ that shows no discrimination (i.e., with a mean of 0.00 and an s.e.m. of 0.07$)$ indicates an even stronger effect. When a $\mathrm{d} 2$ index shows both a difference from zero and a between-group effect (as indicated by ANOVA or paired-samples $t$-statistics), it is called a full effect ${ }^{35}$.

Side preference (or lateralization) of a cohort of animals can be checked for by comparing the amount of time an animal spent exploring the left and the right objects during T1. Similarly, it can be checked whether there is side preference with regard to forward and backward displacement of the moved object in T2 (so a preference for forward- or backward-moved objects). These separate exploration times should not differ too much. One approach to check for side preference is to calculate the difference between the exploration times of the left and right (or forward- and backward-displaced) objects during T1 (i.e., (a1-a2)) or T2, respectively. Subsequently, a $t$ test can be performed in which the difference scores between al and a 2 are compared with the value 0 . In addition, when using an automated tracking program, the amount of time an animal spends in each half (or even each quarter) of the testing arena can be assessed to check for possible area preferences/ avoidances or lateralization, during both T1 and T2. There should be no preference for any specific part of the testing arena during T1. During T2, it is to be expected that an animal shows preference for the specific part of the testing arena to which an object is moved (and identified as moved) and, hence, checking for side preference in T2 is less informative.

\section{Expected results}

When the herein-described procedures for OPS testing (Fig. 3) are applied to naive adult male Wistar rats or $\mathrm{C} 57 \mathrm{BL} / 6$ mice, gradual pattern separation performance effects (spatial discrimination) over 
the different positions should occur (Fig. 2). When all procedural steps are carried out successfully, a graph as shown in Fig. 4 should follow. Figure 4 shows a linear relationship of the discrimination index ( $\mathrm{d} 2$ ) between the different positions. Because the distance of the moved object, as compared with the stationary object, increases, the pattern separation performance (i.e., the $\mathrm{d} 2$ index) increases as well. Usually, rodents show a good pattern separation performance when the displacement is maximal (i.e., position 5) and a 1-h retention interval is interposed between the trials. However, when the displacement of the object along a vertical axis is between the starting position (position 1) and the maximum position (position 5), the pattern separation performance is attenuated. This indicates that it is more challenging for rodents to discriminate between the locations of the objects when the displaced object is closer to its starting position. In other words, a greater distance between the starting location and the new location leads to a better pattern separation performance of the rodents. It is easier to discriminate or perceive the new position as novel when the change is more apparent.

In our laboratory, we mainly use the 1-h inter-trial interval between T1 and T2 in the OPS task. We have found that an inter-trial interval of $2 \mathrm{~h}$ leads to similar results at position 3 in untreated mice $^{15}$. Time-dependent forgetting can be manipulated with experimental conditions, if desired. Although a linear relationship (indicating gradual pattern separation performance effects) has always been observed when performing the OPS task, we have found small sporadic differences in the results for mice and rats at position 3 with regard to the statistical outcome of the post hoc $t$ tests performed after an ANOVA effect. To elaborate, OPS performance at position 3, as compared with the performance at position 1 , has led to a significant effect for rats $(P<0.05)$, but not for mice in the studies described in van Hagen et al. ${ }^{15}$. Both rats and mice showed a significant difference from zero (i.e., chance level, $(P<0.05)$ ), indicating an intermediate effect, and as such, recognition of the familiar object location, implying functioning pattern separation. In both our rat and mouse studies, we have encountered this phenomenon (i.e., position 3 not always reaching statistical significance when compared with position 1) and, as such, we do not assign this performance to a specific species characteristic. The reason for this discrepancy is evident; it results from the fact that position 3 is the cutoff distance at which rodents are just able to differentiate a new spatial arrangement from an earlier encountered one. When using this smallest possible displacement at which the animals are able to discriminate between the 'old' (learning trial) and 'new' (test trial) situation, it is ensured that the presented locations are highly similar to the rodents, and thus pattern separation processes must be used in order to make this discrimination. Inherent to this, the discrimination performance is still low, so it provides the opportunity to measure spatial pattern separation bidirectionally (i.e., it enables measurement of an improvement or an impairment of the OPS performance after an experimental intervention, to resemble the performance at position 5 (e.g., $\mathrm{d} 2$ value increases from 0.15 to 0.30 ) or position 1 (e.g., d2 value decreases from 0.15 to 0.00 ) under naive conditions, respectively). Because the s.e.m. can fluctuate between animal cohorts, and such a refined displacement is used, these slight differences between animal cohorts can occur. Using post hoc $t$ tests that do not perform all pairwise comparisons but instead compare only to the control condition (i.e., position 1, using, e.g., Dunnett's tests) could help to prevent this. Results at position 3 should always be at least statistically different from chance level (i.e., zero) to allow measurement of improvement or impairment after an experimental intervention.

We have successfully used the OPS task for assessing drug effects on pattern separation performance in male Wistar rats ${ }^{11}$. Because intact hippocampal DG functioning is important for proper pattern separation performance ${ }^{4}$, currently, we are investigating the role of adult neurogenesis in the DG in OPS performance. Preliminary results indicate that an increase in adult neurogenesis in the DG, due to a pharmacological manipulation, leads to improved performance in the OPS paradigm. This is in accordance with current hypotheses that adult neurogenesis is important in spatial pattern separation processes. In addition, we are examining the potential of our OPS paradigm for evaluating pattern separation performance in aged rats. For these studies, we used 2-year-old male Wistar rats. Our preliminary findings indicate full applicability of aged rats in the OPS task using a 1-h inter-trial interval and a 3-min trial duration. The group of aged animals shows deficits in OPS performance, which can be attenuated with cognition-enhancing drugs.

In conclusion, the procedures reported in this protocol should provide a framework for assessing spatial pattern separation performance using the OPS task in different experimental conditions. The OPS task solely relies on the natural tendency of rodents to explore novelty and as such does not require punishment or reward incentives or food restriction. The OPS paradigm can be easily implemented in any standard rodent behavioral testing facility by personnel with conventional rodent behavioral expertise. The OPS task is not expensive to incorporate into the behavioral test battery of 
any animal facility already performing rodent behavioral tasks. Especially when ORT or OLT apparatus and experience are available, the OPS task is relatively easy to implement and perform. The OPS task can be a valuable add-on in test batteries when validating new animal models of disease or when investigating novel treatment interventions. Moreover, pattern separation has been linked to neuronal plasticity, including adult-born neurogenesis ${ }^{1}$, opening possibilities for utilization in this field of research as well. The OPS task could allow for not only quick, easy and more comparable research in the field of psychopathology, including studies of MCI, dementia, PTSD and schizophrenia, but also research in the more fundamental neurosciences investigating plasticity or memory formation.

\section{References}

1. Clelland, C. et al. A functional role for adult hippocampal neurogenesis in spatial pattern separation. Science 325, 210-213 (2009).

2. Kheirbek, M. A., Klemenhagen, K. C., Sahay, A. \& Hen, R. Neurogenesis and generalization: a new approach to stratify and treat anxiety disorders. Nat. Neurosci. 15, 1613-1620 (2012).

3. Reagh, Z. M. et al. Spatial discrimination deficits as a function of mnemonic interference in aged adults with and without memory impairment. Hippocampus 24, 303-314 (2013).

4. Schreiber, R. \& Newman-Tancredi, A. Improving cognition in schizophrenia with antipsychotics that elicit neurogenesis through 5- $\mathrm{HT}_{1 \mathrm{~A}}$ receptor activation. Neurobiol. Learn. Mem. 110, 72-80 (2014).

5. Das, T., Ivleva, E. L., Wagner, A. D., Stark, C. E. L. \& Tamminga, C. A. Loss of pattern separation performance in schizophrenia suggests dentate gyrus dysfunction. Schizophr. Res. 159, 193-197 (2014).

6. Tamminga, C. A., Stan, A. D. \& Wagner, A. D. The hippocampal formation in schizophrenia. Am. J. Psychiatry 167, 1178-1193 (2010).

7. Bekinschtein, P. et al. BDNF in the dentate gyrus is required for consolidation of 'pattern-separated' memories. Cell Rep. 5, 759-768 (2013).

8. Oomen, C. A. et al. The touchscreen operant platform for testing working memory and pattern separation in rats and mice. Nat. Protoc. 8, 2006-2021 (2013).

9. Sahay, A., Wilson, D. A. \& Hen, R. Pattern separation: a common function for new neurons in hippocampus and olfactory bulb. Neuron 70, 582-588 (2011).

10. Lacy, J. W., Yassa, M. A., Stark, S. M., Muftuler, L. T. \& Stark, C. E. L. Distinct pattern separation related transfer functions in human CA3/dentate and CA1 revealed using high-resolution fMRI and variable mnemonic similarity. Learn. Mem. 18, 15-18 (2011).

11. van Goethem, N. P., Schreiber, R., Newman-Tancredi, A., Varney, M. \& Prickaerts, J. Divergent effects of the "biased", 5- $\mathrm{HT}_{1 \mathrm{~A}}$ receptor agonists F15599 and F13714 in a novel object pattern separation task. Br. J. Pharmacol. 172, 2532-2543 (2015).

12. Ennaceur, A. \& Delacour, J. A new one-trial test for neurobiological studies of memory in rats. 1: behavioral data. Behav. Brain Res. 31, 47-59 (1988).

13. Leger, M. et al. Object recognition test in mice. Nat. Protoc. 8, 2531-2537 (2013).

14. Antunes, M. \& Biala, G. The novel object recognition memory: neurobiology, test procedure, and its modifications. Cogn. Process 13, 93-110 (2012).

15. van Hagen, B. T. J., van Goethem, N. P., Lagatta, D. C. \& Prickaerts, J. The object pattern separation (OPS) task; a behavioral paradigm derived from the object recognition task. Behav. Brain Res. 285, 44-52 (2015).

16. van Goethem, N. P. et al. Object recognition testing: rodent species, strains, housing conditions, and estrous cycle. Behav. Brain Res. 232, 323-334 (2012).

17. Heyser, J. \& Chemero, A. Novel object exploration in mice: not all objects are created equal. Behav. Process. 89, 232-238 (2012).

18. Prusky, G. T., Harker, K. T., Douglas, R. M. \& Whishaw, I. Q. Variation in visual acuity within pigmented, and between pigmented and albino rat strains. Behav. Brain Res. 136, 339-348 (2002).

19. Roedel, A., Storch, C., Holsboer, F. \& Ohl, F. Effects of light or dark phase testing on behavioural and cognitive performance in DBA mice. Lab. Anim. 40, 371-381 (2006).

20. Akkerman, S. et al. PDE5 inhibition improves object memory in standard housed rats but not in rats housed in an enriched environment: implications for memory models? PLoS ONE 9, e111692 (2014).

21. Bartolomucci, A. et al. Individual housing induces altered immuno-endocrine responses to psychological stress in male mice. Pyschoneuroendocrinology 28, 540-558 (2003).

22. Arndt, S. S. et al. Individual housing of mice - impact on behaviour and stress responses. Physiol. Behav. 97, 385-393 (2009).

23. Castelhano-Carlos, M. J. \& Baumans, V. The impact of light, noise, cage cleaning and in-house transport on welfare and stress of laboratory rats. Lab. Anim. 43, 311-327 (2009).

24. Ameen-Ali, K. E., Eacott, M. J. \& Easton, A. A new behavioural apparatus to reduce animal numbers in multiple types of spontaneous object recognition paradigms in rats. J. Neurosci. Methods 211, 66-76 (2012).

25. Ameen-Ali, K. E., Easton, A. \& Eacott, M. J. Moving beyond standard procedures to assess spontaneous recognition memory. Neurosci. Biobehav. Rev. 53, 37-51 (2015).

26. Albasser, M. M. et al. New behavioral protocols to extend our knowledge of rodent object recognition memory. Learn. Mem. 17, 407-419 (2010). 
27. Kinnavane, L., Albasser, M. M. \& Aggleton, J. P. Advances in the behavioural testing and network imaging of rodent recognition memory. Behav. Brain Res. 285, 67-78 (2015).

28. Sorge, R. E. et al. Olfactory exposure to males, including men, causes stress and related analgesia in rodents. Nat. Methods 11, 629-632 (2014).

29. Ennaceur, A. One-trial object recognition in rats and mice: methodological and theoretical issues. Behav. Brain Res. 215, 244-254 (2010).

30. Akkerman, S. et al. Methodological considerations on discrimination and exploration measures in object recognition. Behav. Brain Res. 232, 335-347 (2012).

31. Bruno, O. et al. GEBR-7b, a novel PDE4D selective inhibitor that improves memory in rodents at non-emetic doses. Br. J. Pharmacol. 164, 2054-2063 (2011).

32. Vanmierlo, T. et al. Liver $\mathrm{X}$ receptor activation restores memory in aged $\mathrm{AD}$ mice without reducing amyloid. Neurobiol. Aging 32, 1262-1272 (2009).

33. Zeef, D. H. et al. Memory deficits in the transgenic rat model of Huntington's disease. Behav. Brain Res. 227, 194-198 (2012).

34. Rutten, K. et al. Automated scoring of novel object recognition in rats. J. Neurosci. Methods 171, 72-77 (2008).

35. Akkerman, S., Prickaerts, J., Steinbusch, H. W. M. \& Blokland, A. Object recognition testing: statistical considerations. Behav. Brain Res. 232, 317-322 (2012).

\section{Acknowledgements}

N.P.v.G. is financially supported by Alzheimer Nederland (grant no. WE.03-2017-11).

\section{Author contributions}

N.P.v.G. and J.P. designed the general OPS procedure for measuring pattern separation performance in rodents. B.T.J.v.H. and N.P.v.G. performed the experiments and analyzed the data. N.P.v.G. and B.T.J.v.H wrote the manuscript with input from J. P.

\section{Competing interests}

The authors declare no competing interests.

\section{Additional information}

Supplementary information is available for this paper at https://doi.org/10.1038/s41596-018-0013-x.

Reprints and permissions information is available at www.nature.com/reprints.

Correspondence and requests for materials should be addressed to J.P.

Publisher's note: Springer Nature remains neutral with regard to jurisdictional claims in published maps and institutional affiliations.

Published online: 23 July 2018

\section{Related links}

Key references using this protocol

1. van Hagen, B. T. J. et al. Behav. Brain Res. 285, 44-52 (2015): https://doi.org/10.1016/j.bbr.2014.10.041

2. van Goethem, N. P. et al. Br. J. Pharmacol. 172, 2532-2543 (2015): https://doi.org/10.1111/bph.13071. 